\title{
Detection of Tumor DNA in Human Plasma with a Functional PLL- Based Surface Layer and Plasmonic Biosensing
}

\author{
Noemi Bellassai, Roberta D’Agata, Almudena Marti, Andrea Rozzi, Stefano Volpi, Matteo Allegretti, \\ Roberto Corradini, Patrizio Giacomini, Jurriaan Huskens,* and Giuseppe Spoto*
}

Cite This: ACS Sens. 2021, 6, 2307-2319

Read Online

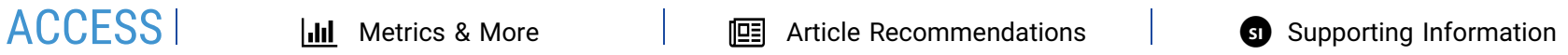

ABSTRACT: Standard protocols for the analysis of circulating tumor DNA (ctDNA) include the isolation of DNA from the patient's plasma and its amplification and analysis in buffered solutions. The application of such protocols is hampered by several factors, including the complexity and time-constrained preanalytical procedures, risks for sample contamination, extended analysis time, and assay costs. A recently introduced nanoparticle-enhanced surface plasmon resonance imaging-based assay has been shown to simplify procedures for the direct detection of tumor DNA in the patient's plasma, greatly simplifying the cumbersome preanalytical phase. To further simplify the protocol, a new dual-functional low-

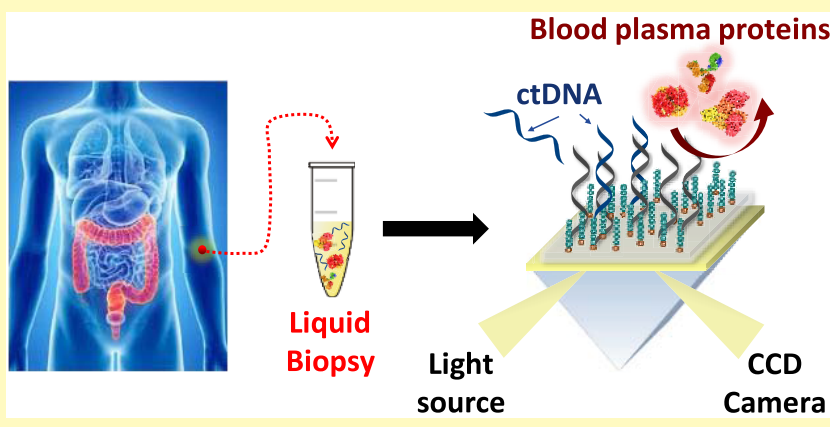
fouling poly-L-lysine (PLL)-based surface layer has been introduced that is described herein. The new PLL-based layer includes a densely immobilized CEEEEE oligopeptide to create a charge-balanced system preventing the nonspecific adsorption of plasma components on the sensor surface. The layer also comprises sparsely attached peptide nucleic acid probes complementary to the sequence of circulating DNA, e.g., the analyte that has to be captured in the plasma from cancer patients. We thoroughly investigated the contribution of each component of the dual-functional polymer to the antifouling properties of the surface layer. The low-fouling property of the new surface layer allowed us to detect wild-type and KRAS p.G12D-mutated DNA in human plasma at the attomolar level ( 2.5 aM) and KRAS p.G13D-mutated tumor DNA in liquid biopsy from a cancer patient with almost no preanalytical treatment of the patient's plasma, no need to isolate DNA from plasma, and without PCR amplification of the target sequence.

KEYWORDS: poly-L-lysine, surface plasmon resonance, cancer diagnosis, plasmonics, peptide nucleic acids

$\mathrm{P}$ recision oncology aims at developing treatments that target personalized tumor drivers, e.g., genomic alterations that usually differ from patient to patient. Since tumors evolve, both spontaneously and under therapeutic pressure, cancer drivers change in space and time, making it mandatory to profile cancer through disease stages and therapeutic settings longitudinally. ${ }^{1}$ This has been done for decades with the carcinoembryonic antigen (CEA) and other conventional biomarkers, but liquid biopsy (LB) holds great promise for the straightforward implementation of precision oncology in cancer treatment. ${ }^{2}$ LB provides a minimally invasive approach to detecting circulating tumor cells, circulating tumor DNA (ctDNA), exosomes, and other actionable biomarkers that freely circulate in body fluids of cancer patients, including blood, urine, saliva, and cerebrospinal fluid. ${ }^{3,4}$ In particular, the clinical management of cancer patients has been shown to benefit from the profiling of ctDNA and cell-free DNA (cfDNA) available in the plasma obtained from patients. ${ }^{5}$ Standard protocols for the analysis of ctDNA include the isolation of DNA from the patient's plasma and its sequencespecific amplification and analysis in buffered solutions. ${ }^{6,7}$ The application of such protocols is hampered by several factors, including the complex preanalytical procedures, the need to process or otherwise stabilize self-decomposing analytes immediately, risks for sample contamination (which increases proportionally to the number of manipulations required), extended analysis time, and consequently assay costs. ${ }^{8}$ Digital bioassays $^{9}$ such as digital PCR ( $\mathrm{dPCR}$ ) have been used to achieve the ultrasensitive detection required to quantify ctDNA in plasma samples with a calibration-free approach and, for this reason, represent the gold standard in current technologies for ctDNA detection in liquid biopsy. However, the obligate preanalytical steps, including cfDNA isolation, and the time-consuming analytical process $(2-4 \mathrm{~h})$ represent

Received: February 19, 2021

Accepted: May 13, 2021

Published: May 25, 2021 
important limitations for the large-scale implementation of similar bioassays for liquid biopsy analysis.

In contrast, biosensors provide a different approach that may bypass several bottlenecks of current molecular diagnoses. Electrochemical biosensing platforms combined with nanostructured materials have been recently shown to hold promise for simple and direct detection of tumor DNA in the serum ${ }^{10}$ or plasma samples. ${ }^{11}$

When circulating cancer biomarkers contained in a complex biological matrix such as human plasma are revealed directly (i.e., without isolation of the biomarker from the blood before the analysis), the nonspecific adsorption of plasma proteins on the active surface of the sensor may negatively interfere with the biosensing performance by preventing the detection of target biomolecules at low concentrations. ${ }^{12}$ Therefore, while promising, biosensors need improvement. Particularly, their surface architecture requires advanced functionalization, and these should ideally introduce at the same time into the surface layer suitable molecular probes for analyte capture and enhanced antifouling properties. A design combining these two features may be regarded as ideal because it helps minimize nonspecific adsorption of components from the biofluids while maintaining the analytical performances of the biosensing platforms. ${ }^{13,14}$

A recent nanoparticle-enhanced surface plasmon resonance imaging (SPRI)-based assay introduced significant improvements to the direct detection of tumor DNA in the patient's plasma. ${ }^{15}$ The assay is based on a sandwich detection approach. The volume of plasma sample used for the analysis is a factor of five times smaller than that used by nextgeneration sequencing (NGS) and $\mathrm{dPCR}$, offering the possibility to multiplex for many distinct DNA sequences and frequently apply the assay in a molecular diagnosis context, with limited amounts of blood, and in rapidly progressing or fragile cancer patients.

Even though the new nanoparticle-enhanced SPRI assay drastically reduces assay time, streamlines diagnostic workflows, and minimizes the potential risk of sample contamination compared to standard methods for liquid biopsy analysis, it still takes $1.5 \mathrm{~h}$ to digest plasma proteins in the preanalytical step and specific additional treatments of the SPRI sensor surface are required to minimize the impact of fouling due to plasma components on the assay performances.

Here, we show that the use of a newly designed poly-L-lysine (PLL)-based dual-functional surface layer, combining fouling resistance in the presence of immobilized peptide nucleic acid (PNA) probes, further improves the performance of the nanoparticle-enhanced SPRI assay, allowing us to implement a novel, highly improved, and further simplified protocol for the direct detection of tumor-related DNA in plasma samples. In particular, the new protocol allows direct detection of ctDNA in the plasma after simple (four-step workflow) and rapid (10 min after the initial centrifugation step) preanalytical processing, thus obviating cumbersome and time-consuming preanalytical procedures to isolate and concentrate template DNA and to PCR-amplify target sequences. ${ }^{16,17}$ Issues in the preanalytical step and workflow complexity constitute essential barriers for the clinical adoption of liquid biopsy assays, ${ }^{18}$ and the new method introduces significant improvements in this respect. It minimizes the risk of sample contamination and significantly simplifies the workflow for preanalytical processing compared to the state-of-the-art assays taking $115 \mathrm{~min}$ with a multistep workflow involving procedures similar to those included in our four-step workflow. ${ }^{19,20}$ The dPCR and NGS state-of the-art assays dealing with PCR amplification require additional time (between 4 and $18 \mathrm{~h}$ ) and additional steps in the workflow. ${ }^{21-25}$

The biosensor antifouling coating here described does not include blocking additives, such as surfactants, protein, and nonprotein-based reagents, leading to a reduction in the biorecognition activity of the immobilized probes. ${ }^{26}$

Antifouling surface layers can be obtained with various materials, ${ }^{27,28}$ such as poly(ethylene glycol) (PEG), ${ }^{29}$ single amino acids and peptides, ${ }^{30}$ zwitterionic compounds, ${ }^{31}$ mixedcharge polymers, ${ }^{32}$ and hydrogels. ${ }^{33}$ Among these compounds, PLL, a homopolymer based on repeat units of the cationic Llysine amino acid, holds remarkable properties as an antifouling material, including superior biocompatibility, predominant hydrophilicity, and good biodegradability. ${ }^{34}$ At physiological $\mathrm{pH}$, the cationic PLL can be deposited on negatively charged surfaces ${ }^{35,36}$ and allows designing monolayers with different functionalities by ensuring critical control over the biosensing interface features. ${ }^{37,38}$ PLL can be modified with various functional groups by introducing neutral or charged side chains, ${ }^{39-41}$ thus offering the possibility to fabricate multifunctional polymeric structures. The main drawback of multifunctional polymers integrating antifouling materials with recognition elements for target capturing is the limited number of target molecules that can access highly packed brush polymers, thereby hampering the biosensing response. ${ }^{42}$ On the other hand, grafting of the recognition elements onto the antifouling layer may negatively affect the antifouling properties of the final layer. ${ }^{4,44}$

The newly designed dual-functional surface layer is based on a mixed-charge PLL polymer structure with antifouling properties. ${ }^{32}$ It comprises an anionic oligopeptide (CEEEEE) and neutral PNA probe side chains. The immobilized oligopeptides, along with the cationic PLL-based polymer, create an antifouling mixed-charge layer, whereas the attached PNA probes provide the target binding partners for the detection of tumor DNA biomarkers directly in human plasma.

The present study aims to thoroughly investigate with SPRI and human plasma the contribution of each component of the dual-functional polymer to the surface layer's antifouling properties. We also focused on a nanoparticle-enhanced SPRI assay with a dual-functional surface layer containing modified PLL and capture probes based on peptide nucleic acids (PNAs) discriminating wild-type (WT) and Kirsten rat viral sarcoma (KRAS) p.G12D- and p.G13D-mutated genomic DNA in human plasma. The KRAS oncogene is recurrently mutated in different human cancers, ${ }^{45}$ and its mutational status is associated with resistance to treatment with antibodies to EGFR. KRAS testing is now mandatory for the standard of care of colorectal cancers (CRCs), e.g., speeding up and facilitating this diagnostic activity would be valuable for biotechnologists, molecular pathologists, medical oncologists, public decisors, and, obviously, patients. Minimal preanalytical sample treatments and SPRI sensor surface conditioning protocols were required for the analysis. Moreover, the WT and p.G12D-mutated genomic DNA in plasma were detected at the $5 \mathrm{pg} \mu \mathrm{L}^{-1}(\sim 2.5 \mathrm{aM})$ level together with p.G13Dmutated ctDNA in a liquid biopsy from a CRC patient, specifically with no previous DNA isolation from plasma and PCR amplification. 


\section{EXPERIMENTAL SECTION}

Chemicals. Reagents were obtained from commercial suppliers and used without further purification. Phosphate-buffered saline tablets (PBS, pH 7.4), poly-L-lysine $\cdot \mathrm{HBr}$ (PLL·HBr) (15-30 kDa), Fmoc-Glu( $\mathrm{OtBu})$-Wang resin, trifluoroacetic acid (TFA), triisopropylsilane (TIS), diethyl ether, hydroxybenzotriazole hydrate (HOBt), 2-(1H-benzotriazole-1-yl)-1,1,3,3-tetramethyluronium hexafluorophosphate (HBTU), N,N-diisopropylethylamine (DIPEA), $\mathrm{N}$-methyl-2-pyrrolidone (NMP), piperidine, dichloromethane (DCM), and methanol were purchased from Sigma-Aldrich (The Netherlands). Fmoc-protected PNA monomers and the SPDP-(PEG) ${ }_{4}$ spacer were purchased from LGC-Link (U.K.), and resin for PNA synthesis (Rink Amide ChemMatrix) was purchased from Sigma-Aldrich (Italy). Trisodium citrate dihydrate, tetrachloroauric(III) acid, ethanol, dimethyl sulfoxide, and sodium hydroxide solutions (10 $\mathrm{M}$ in water) were purchased from Sigma-Aldrich (Italy). (NHS)-Tetra(ethylene glycol)-maleimide (NHS-(EG) 4 -mal) and Zeba Spin Desalting Columns (7 kD MWCO, $5 \mathrm{~mL}$ ) were purchased from Thermo Fischer Scientific (The Netherlands). Tris(2-carboxyethyl)phosphine (TCEP) disulfide reducing gel was purchased from Thermo Fischer Scientific (Italy). The oligopeptide CEEEEE ( $>95 \%$ purity grade; MW: $766.73 \mathrm{~g} \mathrm{~mol}^{-1}$ ) was obtained by Selleck Chemicals LLC (Europe). Pooled human plasma from healthy donors (SER-PLE human recovered plasma frozen; anticoagulant: EDTA 200 $\mathrm{mL}$ ) was purchased from ZenBio (North Carolina). Phosphatebuffered saline (PBS) solutions at pH $7.4(137 \mathrm{mM} \mathrm{NaCl}, 2.7 \mathrm{mM}$ $\mathrm{KCl}$, phosphate buffer $10 \mathrm{mM}$ ) were obtained from VWR (Italy). Wild-type streptavidin (WT-SA) was obtained from Invitrogen (Italy). Nitrocellulose membrane filters were purchased from Whatman (U.K.). Gold chips were purchased from Xantec bioanalytics GmbH (Germany). N- $\alpha$-Fmoc-L-glutamic acid $\gamma$-t.-butyl ester (Fmoc-Glu(OtBu)-OH) was purchased from Merk Millipore (The Netherlands). Multisyntech $\mathrm{GmbH}$ instrument was employed for the automated solid-phase peptide synthesis (SPPS) of the oligopeptide. Ultrapure water (Milli-Q Element, Millipore) was used for all experiments.

PNA Probes. We designed PNA probes for wild-type, p.G12D, and p.G13D KRAS sequences to obtain high melting temperatures (higher than $65^{\circ} \mathrm{C}$ at $4 \mu \mathrm{M}$ concentration), as calculated according to an empirical model ${ }^{46}$ with full match complementary DNA sequence. Melting temperatures were estimated for $4 \mu \mathrm{M}$ concentration using the online available PNA design tool from PNA BIO (http://pnabio. com/support/PNA Tool.htm) along with Giesen et al. ${ }^{46}$ Table 1 shows sequences of the designed and synthesized PNA probes and their acronym.

Table 1. Sequences and Acronyms of PNA Probes Used for SPR Experiments ${ }^{a}$

\begin{tabular}{|c|c|c|c|}
\hline gene, exon & $\begin{array}{l}\text { mutational } \\
\text { status }\end{array}$ & PNA probe sequence & acronym \\
\hline \multirow[t]{3}{*}{$\begin{array}{l}\text { KRAS } \\
\text { exon } 2\end{array}$} & wild-type & $\begin{array}{l}\text { SPDP-(PEG) })_{4-}^{-} \\
\text {CTACGCCACCAGCT-Gly-NH }\end{array}$ & PNA-WT \\
\hline & p.G12D & $\begin{array}{l}\text { SPDP-(PEG) } \text { S- }^{-} \\
\text {CTACGCCATCAGCT-Gly-NH }\end{array}$ & $\begin{array}{l}\text { PNA- } \\
\text { G12D }\end{array}$ \\
\hline & p.G13D & $\begin{array}{l}\text { SPDP-(PEG)4- } \\
\text { CTACGTCACCAGCT-Gly-NH2 }\end{array}$ & $\begin{array}{l}\text { PNA- } \\
\text { G13D }\end{array}$ \\
\hline
\end{tabular}

${ }^{a}$ SPDP, N-succinimidyl 3-(2-pyridyldithio)propionate; PEG, poly(ethylene glycol).

We synthesized PNA probes by automatic solid-phase synthesis and purified and characterized them as described elsewhere ${ }^{47}$ and briefly reported in the Supporting Information (Figures S2-S5). Protected thiol moieties were introduced in PNA-WT and PNAG12D probes using commercially available SPDP-(PEG)4-acid formed of 3-(2-pyridyldithio)propionate (SPDP) and four ethylene glycol units that operated as spacers for avoiding steric hindrance to the access to PNA probes (PNA-WT MW: $4219 \mathrm{~g} \mathrm{~mol}^{-1}$; PNA-G12D MW: $4233 \mathrm{~g} \mathrm{~mol}^{-1}$; PNA-G13D MW: $\left.4233 \mathrm{~g} \mathrm{~mol}^{-1}\right)$. We deprotected thiol groups in PNA-WT and PNA-G12D before their use through TCEP disulfide reducing gel. With this aim, $40 \mu \mathrm{L}$ of TCEP disulfide reducing gel was centrifuged $(3300 \mathrm{rpm}, 1 \mathrm{~min}, 25$ ${ }^{\circ} \mathrm{C}$ ) and the supernatant was removed and discarded. The procedure was repeated twice and then the gel was washed by adding $20 \mu \mathrm{L}$ of PBS buffer, briefly vortexed to resuspend the gel, and centrifuged (3300 rpm, $1 \mathrm{~min}, 25{ }^{\circ} \mathrm{C}$ ). The supernatant was removed and discarded. Then, $20 \mu \mathrm{L}$ of PNA stock solution was added in two tubes containing the treated gel, vortexed, and incubated for $15 \mathrm{~min}$ at 25 ${ }^{\circ} \mathrm{C}$. After the centrifugation at $25{ }^{\circ} \mathrm{C}$ for $1 \mathrm{~min}$, the supernatant containing the thiol-PNA probe was collected. The final concentration of the thiol-PNA solution was determined by UV-vis spectroscopy (Figure S6 and Table S1).

Synthesis and Functionalization of Gold Nanoparticles. All glassware we used to synthesize and store AuNPs were cleaned with aqua regia $\left(3: 1 \mathrm{HCl} / \mathrm{HNO}_{3}\right)$ and then with freshly prepared piranha solution $\left(1: 3\right.$ mixture of $30 \% \mathrm{H}_{2} \mathrm{O}_{2}$ and $\left.96 \% \mathrm{H}_{2} \mathrm{SO}_{4}\right)$ and thoroughly rinsed with ultrapure $\mathrm{H}_{2} \mathrm{O}$. We synthesized AuNPs by citrate reduction of $\mathrm{HAuCl}_{4} \cdot 3 \mathrm{H}_{2} \mathrm{O}$ according to the method elsewhere described. ${ }^{48}$ Briefly, $20 \mathrm{~mL}$ of trisodium citrate $(38.8 \mathrm{mM})$ was quickly added with vigorous stirring to $200 \mathrm{~mL}$ of a boiling solution of $\mathrm{HAuCl}_{4} \cdot 3 \mathrm{H}_{2} \mathrm{O}(1 \mathrm{mM})$. The color of the solution changed from pale yellow to deep red in a few seconds. A complete reduction of trisodium citrate was obtained after 6-8 min upon boiling. The solution was cooled to room temperature and filtered through a 0.45 $\mu \mathrm{m}$ mixed-cellulose ester membrane filter. Colloidal gold dispersions were stored in the dark and refrigerated at $4{ }^{\circ} \mathrm{C}$. Similar conditions assured nanoparticle stability for several months.

The AuNP dispersion was passivated with poly(vinylpyrrolidone) (PVP) as previously reported ${ }^{15}$ and modified with streptavidin (SA) and then with a biotinylated oligonucleotide sequence (DNA sequence: $5^{\prime}$-CAAGTTTATATTCAGTCAT- $3^{\prime}$ ). Briefly, $500 \mu \mathrm{L}$ of citrate-stabilized AuNPs ( $5 \mathrm{nM}$ in water) was added to $500 \mu \mathrm{L}$ of PVP (50 $\mu \mathrm{M}$ in water). The resulting solution was rotated on a thermomixer for $16-18 \mathrm{~h}\left(300 \mathrm{rpm}\right.$ at $\left.23^{\circ} \mathrm{C}\right)$. After the separation of the liquid phase by centrifugation $\left(30 \mathrm{~min}, 13500 \mathrm{rpm}\right.$ at $23^{\circ} \mathrm{C}$ ) to pellet the PVP-modified nanoparticles (AuNP@PVP), they were suspended in $50 \mu \mathrm{L}$ of water. A volume of $25 \mu \mathrm{L}$ of SA solution ( $1 \mathrm{mg}$ $\mathrm{mL}^{-1}$ in water) was added to $100 \mu \mathrm{L}$ of AuNP@PVP solution, and the dispersion was mixed for $1.5 \mathrm{~h}$ on a thermomixer ( $300 \mathrm{rpm}$ at 23 ${ }^{\circ} \mathrm{C}$ ) to obtain the adsorption of SA on passivated gold nanoparticles (AuNP@PVPSA). After discarding the supernatant by centrifugation $\left(20 \mathrm{~min}, 13000 \mathrm{rpm}\right.$ at $23{ }^{\circ} \mathrm{C}$ ) to pellet particles and separate unbound SA, $2 \mu \mathrm{L}$ of biotinylated oligonucleotide (100 $\mu \mathrm{M}$ in water) was combined with $20 \mu \mathrm{L}$ of AuNP@PVPSA diluted to $40 \mu \mathrm{L}$ with water. The dispersion was mixed and rotated for $2.5 \mathrm{~h}$ on a thermomixer $\left(300 \mathrm{rpm}\right.$ at $\left.23{ }^{\circ} \mathrm{C}\right)$. The dispersion was centrifugated for $15 \mathrm{~min}\left(13000 \mathrm{rpm} 23^{\circ} \mathrm{C}\right)$ to pellet the particles and discard the supernatant, and AuNP@KRAS were resuspended in $50 \mu \mathrm{L}$ of water for storage (as the stock solution). The bare and conjugated gold nanoparticles were characterized by UV-vis spectroscopy (VersaWave spectrophotometer, Expedeon Ltd., U.K.), dynamic light scattering, and $\zeta$-potential measurements (Zetasizer Nano ZS ZEN3600, Malvern Instruments, Malvern, U.K.), by diluting the stock solution in PBS buffer $(0.1 \mathrm{nM})$. UV-vis spectra acquired before and after nanoparticles functionalization along with dynamic light scattering and $\zeta$-potential data are shown in Figure S7 and Table $S 2$, respectively.

Surface Plasmon Resonance Imaging (SPRI). We performed SPRI experiments using an SPR imager apparatus (GWC Technologies) and analyzed SPR images using V++ (version 4.0, Digital Optics Limited, New Zealand) and Image J 1.32j (National Institutes of Health) software. SPRI pixel intensity values were converted into the percentage of reflectivity $(\% R)$ using the equation $\% R=100 \times\left(0.85 I_{\mathrm{p}} / I_{\mathrm{s}}\right)$, where $I_{\mathrm{p}}$ and $I_{\mathrm{s}}$ refer to $\mathrm{p}$ - and s-polarized reflected light, respectively. We obtained SPRI kinetic data by plotting the difference in percent reflectivity $(\Delta \% R)$ from selected regions of interest of SPR images as a function of time. All SPRI experiments were performed at room temperature. We used a microfluidic device with six parallel microchannels $(80 \mu \mathrm{m}$ depth, $1.4 \mathrm{~cm}$ length, $400 \mu \mathrm{m}$ 
width) to achieve independent control of parallel interactions occurring on the gold chip surface. The device was fabricated using poly(dimethylsiloxane) (PDMS) and connected with PEEK and Tygon tubes (UpChurch Scientific) to a peristaltic pump (IPC, Ismatec SA, Switzerland).

A cleaning procedure of the fluidic system was implemented to minimize the risk of contaminations and memory effects. In particular, after each experiment, the fluidic system was washed with ultrapure water $\left(37^{\circ} \mathrm{C}\right.$, for $2 \mathrm{~h}$ ) and PBS buffer (at least $1 \mathrm{~h}$ ). Every 3 weeks, the following cleaning protocol for Tygon tubes was applied: $0.5 \%$ sodium dodecyl sulfate (SDS) (10 $\mathrm{min}), 6 \mathrm{M}$ urea (10 $\mathrm{min}), 1 \%$ acetic acid $(10 \mathrm{~min}), 0.2 \mathrm{M} \mathrm{NaHCO}_{3}(10 \mathrm{~min})$, and ultrapure water $\left(37^{\circ} \mathrm{C}, 30 \mathrm{~min}\right)$. Before each experiment, cleaned Tygon tubes were conditioned by flowing PBS buffer at least for $1 \mathrm{~h}$.

Functionalization of the SPRI Sensor Gold Surface. We cleaned the SPRI gold chip with ultrapure water and ethanol and dried it under $\mathrm{N}_{2}$. The chip was then treated with UV-ozone (10 min) and used for SPRI experiments. We synthesized poly-L-lysine-gmaleimide(26\%) (PLL-mal(26\%)) used for chip functionalization by

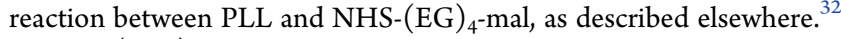
PLL-mal (26\%) was adsorbed on the gold chip surface introducing its solution $\left(0.5 \mathrm{mg} \mathrm{mL}^{-1}\right)$ in PBS into the microfluidic device in contact with the SPRI sensor surface (flow rate $20 \mu \mathrm{L} \mathrm{min}^{-1}, 15 \mathrm{~min}$ ). The surface was then washed with PBS for $10 \mathrm{~min}$ to remove unbound PLL-mal (26\%). We then freshly deprotected the thiolated PNA probes $(0.1 \mu \mathrm{M}$ in PBS) using TCEP gel and coupled them to the maleimide units of the surface-adsorbed PLL-mal $(26 \%)$ by thiolmaleimide Michael-type addition to yield PLL-mal(26\%)-PNA. We obtained the spatially separated immobilization of the probes by introducing PNA-WT, PNA-G12D, and PNA-G13D solutions (0.1 $\mu \mathrm{M}$ in PBS, flow rate $10 \mu \mathrm{L} \mathrm{min}^{-1}$ ) into parallel microchannels of the microfluidic device in contact with the PLL-mal(26\%)-modified SPRI surface. The dual-functional PLL-mal(26\%)-PNA-CEEEEE polymer was obtained by reaction between the thiol moiety in CEEEEE and unreacted maleimide units in PLL-mal(26\%)-PNA. With this aim, a CEEEEE solution (1.0 mM in PBS) was adsorbed on PLL-mal(26\%)PNA (flow rate $10 \mu \mathrm{L} \mathrm{min}{ }^{-1}, 40 \mathrm{~min}$ ).

Antifouling Performance of the Modified Surfaces. We evaluated the antifouling capacity of the surface layers by quantifying the surface coverage of protein adsorbed on the functionalized surfaces after exposure to diluted human plasma (10\% in PBS) using SPRI. Experiments were conducted using diluted pooled human plasma centrifuged at $10800 \mathrm{rpm}$ for $10 \mathrm{~min}$ at $4{ }^{\circ} \mathrm{C}$ and its PBS solutions. First, a stable SPR signal was established by flowing a

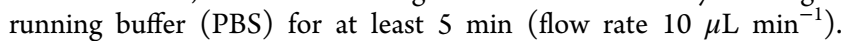
Then, diluted (in PBS) human plasma samples were allowed to flow for $30 \mathrm{~min}$. During the last step of the SPRI experiment, we replaced the human plasma sample with PBS that was flowed for $10 \mathrm{~min}$ to wash off weakly adsorbed protein and to establish the final baseline. We tested the antifouling properties of different surfaces modified with the PLL polymer (namely, PLL, PLL with PNA (PLL/PNA), PLL with PNA and CEEEEE (PLL/PNA/CEEEEE; see Table 2,

Table 2. Surface Coverage $\left(\mathrm{ng} \mathrm{cm}^{-2}\right)$ of Plasma Components on PLL-Based Layers ${ }^{a}$

\begin{tabular}{llc}
\multicolumn{1}{c}{ surface layer } & \multicolumn{1}{c}{ notes } & $\Gamma\left(\mathrm{ng} \mathrm{cm}^{-2}\right)$ \\
PLL & no maleimide linker & $535 \pm 17$ \\
PLL/PNA & no maleimide linker & $558 \pm 54$ \\
PLL/PNA/CEEEEE & no maleimide linker & $347 \pm 45$ \\
PLL-mal(26\%) & no PNA, no CEEEEE & $364 \pm 13$ \\
PLL-mal(26\%)-PNA & no CEEEEE & $381 \pm 31$ \\
PLL-mal(26\%)/EEEEE & no PNA, no Cys & $213 \pm 53$ \\
PLL-mal(26\%)-CEEEEE & no PNA & $48^{32}$ \\
PLL-mal(26\%)-PNA-CEEEEE & dual-functional PLL & $46 \pm 34$
\end{tabular}

${ }^{a}$ The standard deviation refers to three independent experiments. Nine replicates were instead considered for PLL-mal(26\%)-PNACEEEEE.
Figures S8 and 4), and PLL-mal(26\%) (namely, PLL-mal(26\%), PLLmal(26\%) with PNA (PLL-mal(26\%)-PNA), and PLL-mal(26\%)PNA-CEEEEE; see Table 2 and Figure 4)). To assess the electrostatic contribution of the peptide to the antifouling activity, CEEEEE and EEEEE were separately adsorbed on the PLL-mal(26\%) polymer and the relevant antifouling properties were tested as already described. We synthesized the oligopeptide Glu-Glu-Glu-Glu-Glu (or EEEEE) by the SPPS method using the Fmoc-Glu(OtBu)-Wang resin. EEEEE was purified using high-performance liquid chromatography (HPLC) on a Water (2535) setup, equipped with analytical and preparative XBridge $\mathrm{C} 18$ columns, and characterized by mass spectrometry (Figure S1). SPRI kinetic profiles that we detected for the immobilization of the antifouling layers modified with PLL (with and without the maleimide linker) and PLL-mal(26\%) with the EEEEE peptide are shown in Figure S9. We quantified the surface coverage of protein adsorbed on the surface of the treated SPRI chips by measuring the variation of $\Delta \% R$ after the injection of the human plasma sample. The mass of adsorbate per unit area $\left(\Gamma, \mathrm{ng} \mathrm{cm}^{-2}\right)$ was estimated based on the theoretical model described by ShumakerParry et al., ${ }^{49}$ where the specific density for the plasma protein was $\rho_{\mathrm{PP}}$ $=1.42 \mathrm{~g} \mathrm{~cm}^{-3}$, as the average value of the specific density of plasma proteins, ${ }^{50}$ the refractive index of the plasma protein was $n_{\mathrm{PP}}=1.53,{ }^{51}$ and the refractive index of the PBS buffer was 1.33. We also considered the decay length $l_{\mathrm{d}}$ as $37 \%$ of the SPR wavelength. ${ }^{52}$ The sensitivity factor for the SPRI system was $s=6009.28 \% R /$ RIU.

DNA Samples and SPRI Detection. Wild-type genomic DNA (gDNA) was isolated from HT-29 human cells, whereas p.G12Dmutated gDNA was isolated from LS174T cells according to the protocol described elsewhere. ${ }^{53}$ Individual plasma samples were obtained from Regina Elena Institutional BioBank. A stage IV colorectal cancer (CRC) patient and a healthy donor donated the anonymized clinical samples we used for the analyses. gDNAs and cfDNAs were KRAS-genotyped by targeted NGS and dPCR according to protocols described elsewhere. ${ }^{53}$ Plasma sample pt\#34 (CRC patient) contained 12215 copies $\mathrm{mL}^{-1}$ of p.G13D KRASmutated ctDNA and 15547 copies $\mathrm{mL}^{-1}$ of wild-type cfDNA (variant allele frequency 44\%). Plasma sample \#4 (healthy donor) contained $<1$ copies $\mathrm{mL}^{-1}$ of mutated ctDNA and 1514 copies $\mathrm{mL}^{-1}$ of wildtype cfDNA (variant allele frequency $<0.01 \%$ ).

We used the SPRI assay to detect the genetic targets in spiked plasma based on a sandwich detection approach. ${ }^{54}$ PNA probes were covalently bound to PLL-mal $(26 \%)$ previously adsorbed on the surface of the SPRI gold sensor. CEEEEE was then covalently bound to PLL-mal(26\%)-PNA (Figure 1). gDNA in human plasma was then adsorbed on PLL-mal(26\%)-PNA-CEEEEE, leading to the hybridization between the relevant PNA probe and the complementary sequence of the target. We used functionalized AuNPs in the last step of the assay to enhance plasmonic detection. With this aim, we decorated AuNPs with an oligonucleotide whose sequence is complementary to a portion of the sequence of the immobilized target not hybridized with the PNA probe. Plasma samples were first centrifuged at $10800 \mathrm{rpm}\left(4{ }^{\circ} \mathrm{C}\right)$ for $10 \mathrm{~min}$, and then later, the supernatant was diluted in a 1:10 ratio. We detected p.G12D-mutated and wild-type gDNAs spiked in $10 \%$ diluted human plasma at a final concentration of $5 \mathrm{pg} \mu \mathrm{L}^{-1}(\sim 2.5 \mathrm{aM})$.

Before SPRI experiments, we sonicated ( 3 min, ELMA Transsonic T480/H-2) and vortexed (1 min, IKA Vortex GENIUS 3) spiked plasma samples. gDNAs were denatured by heating $\left(95^{\circ} \mathrm{C}\right.$ for $\left.5 \mathrm{~min}\right)$ the spiked plasma sample. Strand reassociation was limited by cooling the treated samples (on ice, $1 \mathrm{~min}$ ) before their introduction into the SPRI microfluidic device $\left(10 \mu \mathrm{L} \mathrm{min}{ }^{-1}\right)$. We loaded wild-type and mutated gDNA plasma samples into nearby channels of the microfluidic device taking care to swap the order of the loaded samples when moving from an experiment to the following one. The same protocol was adopted for the analysis of liquid biopsy from the CRC patient and individual healthy donor. 


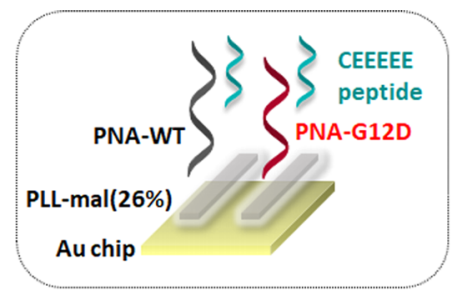

(a)

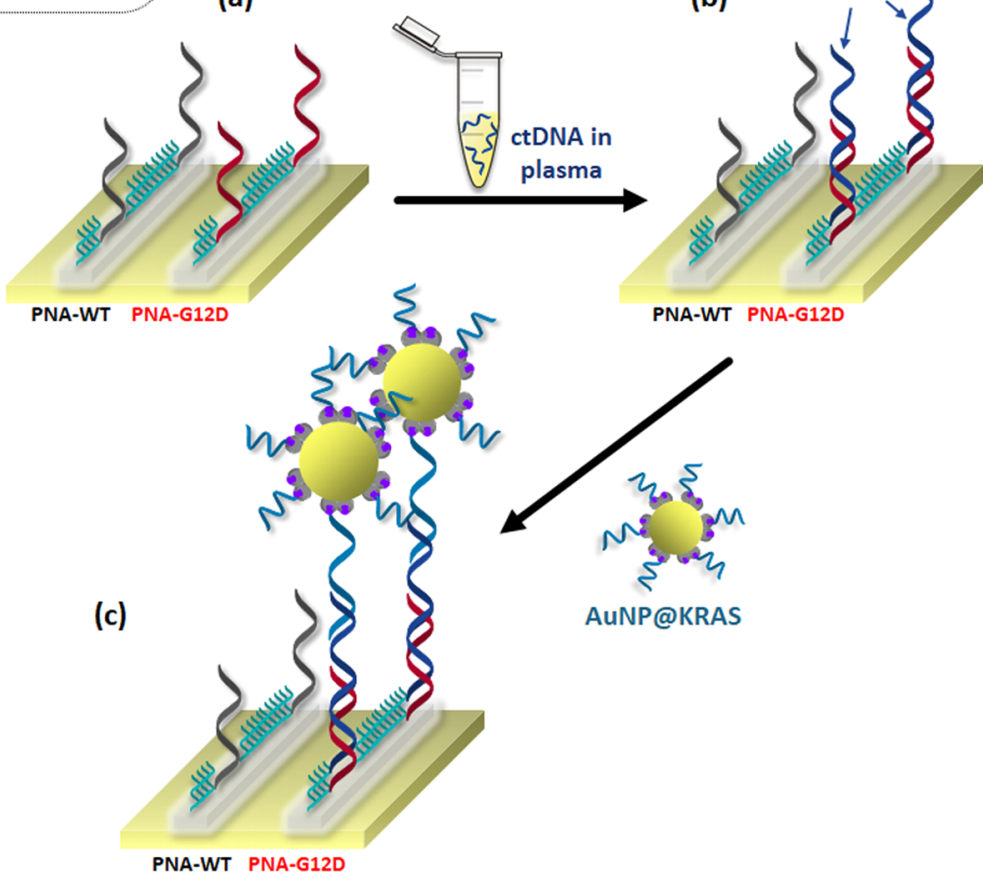

Figure 1. Pictorial representation of the ultrasensitive detection of the p.G12D KRAS-mutated ctDNA sequence in human plasma samples using (a) the dual-functional PLL-based polymer and the nanoparticle-enhanced SPR-based sandwich assay. To simplify the representation, only specifically adsorbed ctDNA is shown. (b) We adsorbed the plasma sample on both PNA-WT and PNA-G12D probes (c) to recognize the mutated DNA sequence and discriminate it from wild-type DNA.

\section{RESULTS AND DISCUSSION}

\section{Dual-Functional PLL-mal(26\%)-PNA-CEEEEE on a} Sensor Surface. Figure 2 summarizes the surface functionalization steps we adopted to develop the dual-functional PLLbased polymer used for the direct SPRI detection of cancer biomarkers in human plasma. The PLL-based polymer includes both an anionic oligopeptide (CEEEEE) and neutral PNA probe side chains. The anionic oligopeptides, along with the cationic PLL-based polymer, provide an antifouling mixedcharge layer able to minimize the unspecific adsorption of components in human plasma on the surface. At the same time, PNA probes provide specific recognition of the analyte.

We adsorbed PLL-mal(26\%) onto the UV-/ozone-treated surface of an SPRI gold sensor by electrostatic interactions (Figure 2a). For this reason, we introduced a PLL-mal(26\%) solution $\left(0.5 \mathrm{mg} \mathrm{mL}^{-1}\right.$ in PBS) into the parallel channels of the microfluidic device in contact with the SPRI gold surface. We then coupled thiolated PNA probes (PNA-WT and PNAG12D, $0.1 \mu \mathrm{M}, 30 \mathrm{~min}$ ) to the maleimide units of PLL$\operatorname{mal}(26 \%)$ (Figure 2b). The parallel microchannels of the device provided the separated immobilization of PNA-WT and PNA-G12D probes. The anionic CEEEEE peptide was lastly anchored to residual maleimide moieties of PLL-mal(26\%)PNA (Figure 2c).

We first covalently immobilized PNA probes on PLL$\mathrm{mal}(26 \%)$ to take under control the probe surface density by monitoring the SPRI kinetic response. The steric hindrance caused by densely immobilized probes may affect the efficiency of the PNA/DNA target hybridization reaction. ${ }^{55}$ In contrast, the immobilized probes' low surface density limits the number of PNA molecules available for DNA interaction. ${ }^{56}$ A PNA surface coverage of $3 \times 10^{12}$ molecules $\mathrm{cm}^{-2}$ has been shown to allow ultrasensitive nanoparticle-enhanced SPRI detection of the DNA target. ${ }^{15}$

Figure 3 shows representative SPRI kinetic responses detected during the surface functionalization steps mentioned above, leading to the formation of the dual-functional PLLmal(26\%)-PNA-CEEEEE system. After each reactive step (i.e., PLL-mal(26\%) adsorption, PNA probe, and CEEEEE immobilization), we washed the surface with PBS to remove unreacted species.

Different kinetic profiles were detected for the immobilization of the three systems composing the functional layer. The PLL-mal(26\%) polymer was immobilized on the activated gold surface with fast adsorption kinetics reaching the signal saturation within a few minutes after the electrostatic interaction between the positively charged polymer and the negatively charged gold surface. By contrast, PNA probes and CEEEEE exploit the covalent coupling between thiol groups and maleimide units exposed by the immobilized PLLmal(26\%). Kinetic SPR profiles referring to such interactions indicate a slower reaction than the polymer adsorption on gold, as reported elsewhere. ${ }^{32}$ As expected, the smaller size and higher concentration of CEEEEE compared with PNA probes 


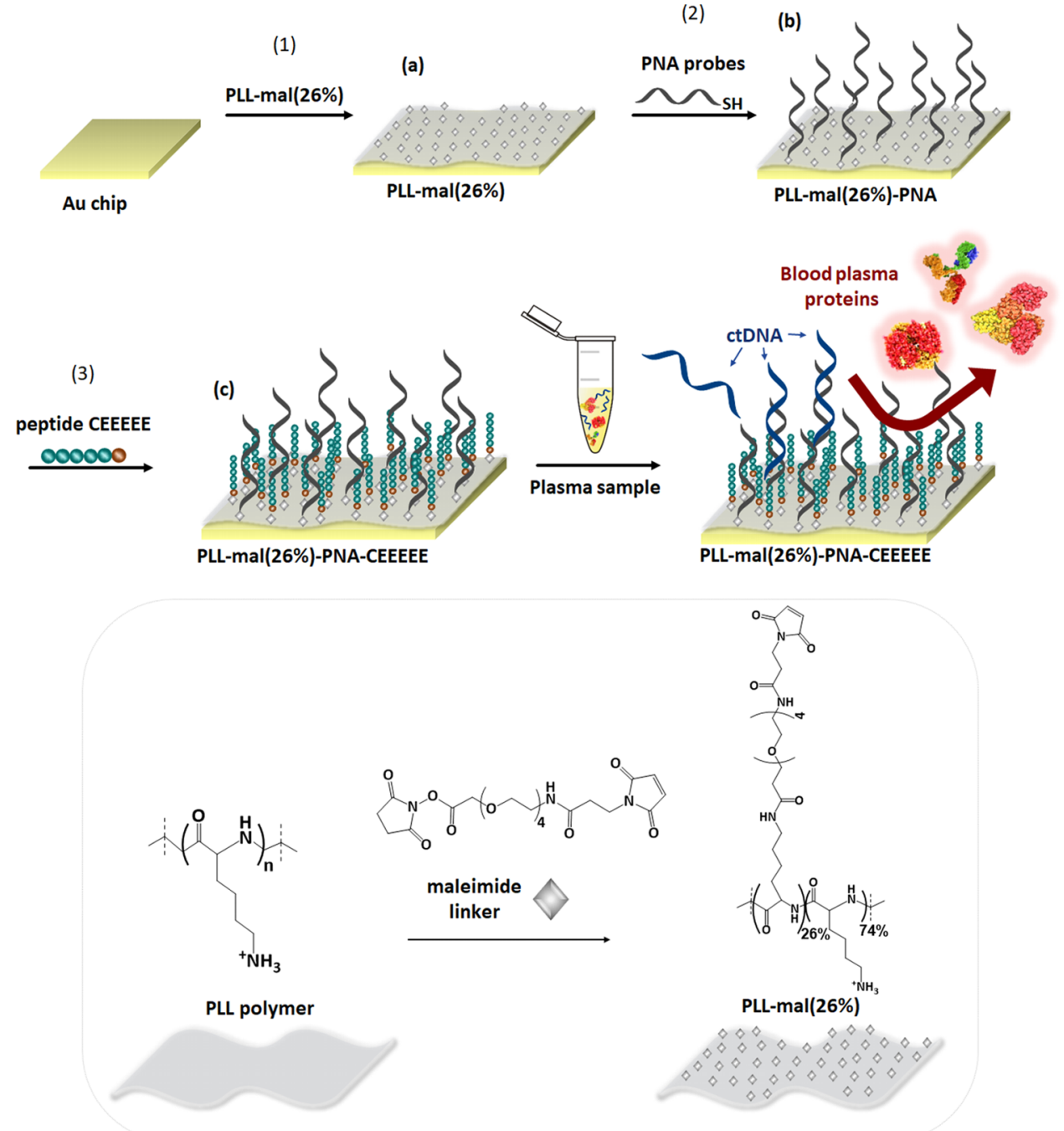

Figure 2. Pictorial representation of (a) PLL-mal(26\%), (b) PNA probe, and (c) CEEEEE immobilization on the surface of the SPR gold sensor for the fabrication of the dual-functional PLL-based surface layer. The exposure of the PLL-mal(26\%)-PNA-CEEEEE final surface to plasma samples allows both the hybridization of the circulating tumor DNA (ctDNA) target with the complementary PNA probe and the repulsion of plasma proteins.

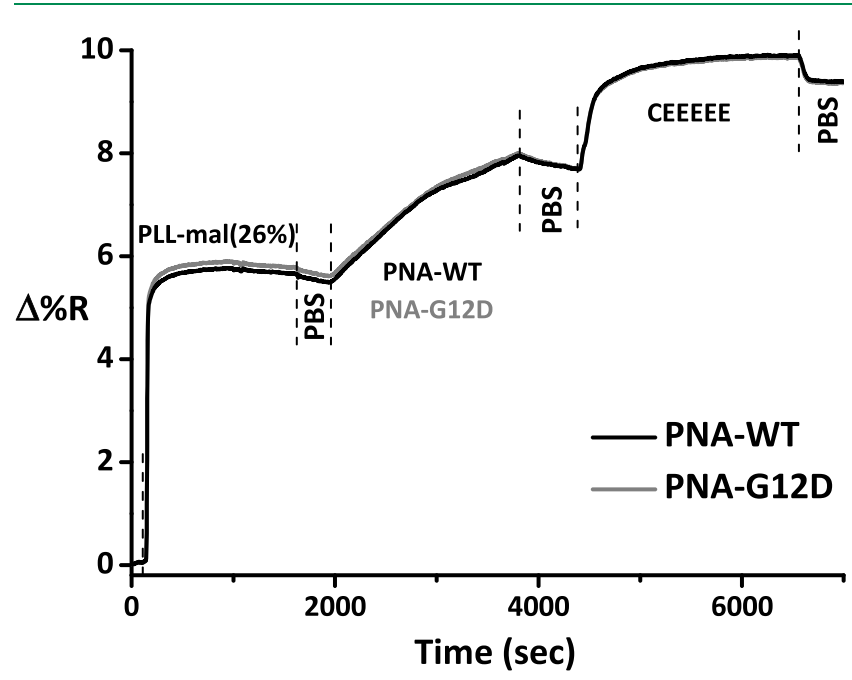

Figure 3. Percent reflectivity $(\Delta \% R)$ over time detected for PLL$\mathrm{mal}(26 \%)$ deposition, PNA-WT or PNA-G12D immobilization (0.1 $\mu \mathrm{M}, 30 \mathrm{~min})$, and CEEEEE (1.0 $\mathrm{mM}, 40 \mathrm{~min})$ anchoring to the PLL$\operatorname{mal}(26 \%)-P N A$ layer. favors its reaction with maleimide, thereby reaching the signal saturation faster than PNAs. The fabrication of the dualfunctional polymer involves consecutive surface functionalization and washing steps with the PBS buffer to remove unreacted species and stabilize the final surface. The last portion of the SPR sensorgram shown in Figure 3 refers to the last PBS washing step. The stable baseline detected during such a step demonstrates the stability of the formed surface layer.

The detected $\Delta \% R$ values, which ultimately depend on the molecular weight of the adsorbed species and on the densities and refractive indexes of both the formed layer and the solution in contact with the surface, allow estimating structural parameters of the adsorbed layer (i.e., thickness and surface coverage) based on the model described by Shumaker-Parry et al. ${ }^{49}$ For the calculation, we considered refractive indexes of PLL-mal $n_{\mathrm{PLL}}=1.52$, PBS buffer $n_{\mathrm{PBS}}=1.33$, CEEEEE $n_{\mathrm{CE}}=$ 1.44 , and PNA probes $n_{\mathrm{PNA}}=1.40$.

The proper immobilization of PNA probes onto the SPR sensor required accurate monitoring of the anchored probes' surface density to ensure the successful detection of the wildtype or p.G12D-mutated DNA targets. It involves comparing 

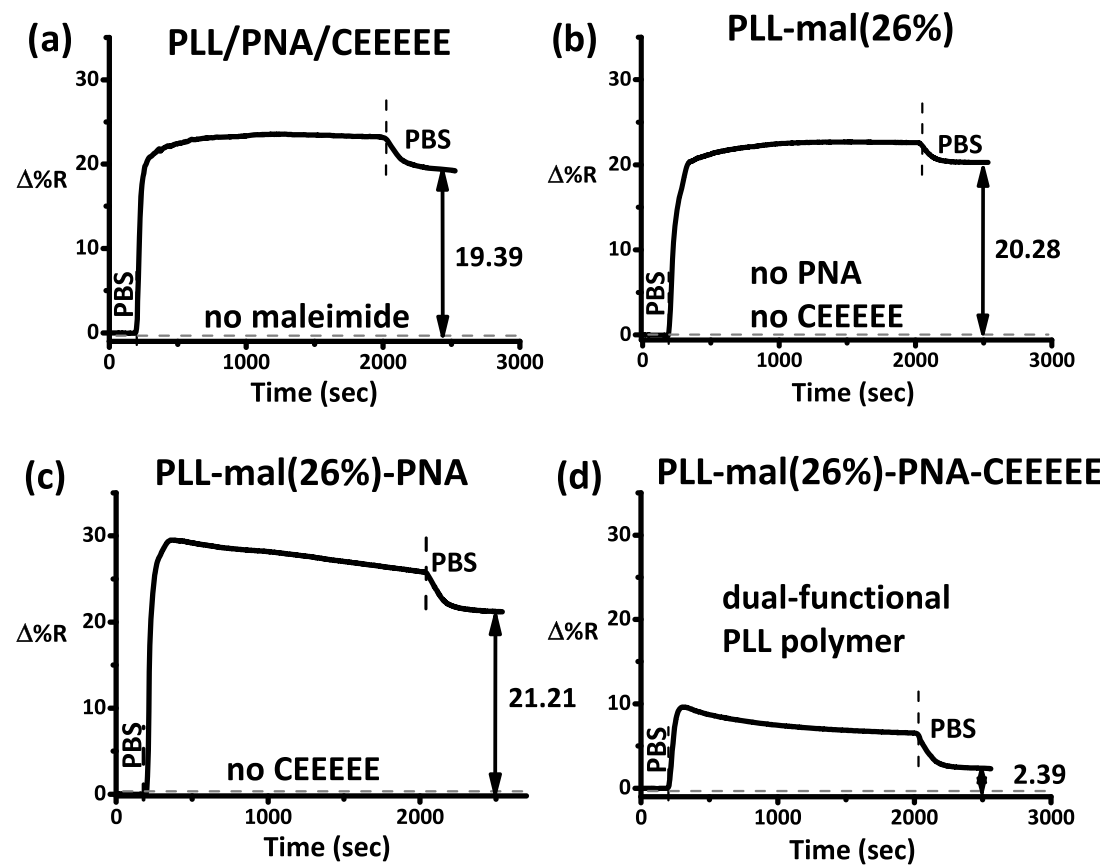

Figure 4. SPRI data for the adsorption of $10 \%$ diluted human plasma (30 min) on (a) PLL/PNA/CEEEEE, (b) PLL/PNA, (c) PLL-mal(26\%), and (d) PLL-mal(26\%)-PNA-CEEEEE layers. PNA-WT was used as the probe.

SPRI signals detected from surfaces where the two probes are immobilized. Figure 3 shows the immobilization of PNA-WT and PNA-G12D probes resulting in similar $\Delta \% R$ values corresponding to $5 \times 10^{12}$ molecules $\mathrm{cm}^{-2}$. The subsequent immobilization of CEEEEE leads to the formation of a more densely packed layer compared to PNA $\left(21 \times 10^{12}\right.$ molecules $\mathrm{cm}^{-2}$ ). The leveling off of the adsorption curve indicates saturation of sites and/or steric maximization of the surface density. The total surface coverage of PNA and CEEEEE obtained here $\left(26 \times 10^{12}\right.$ molecules $\left.\mathrm{cm}^{-2}\right)$ agrees with that estimated based on independent cyclic voltammetry experiments described elsewhere $\left(32 \times 10^{12}\right.$ molecules $\left.\mathrm{cm}^{-2}\right) .{ }^{40}$

Contribution of Individual Components to the Antifouling Performance of the Dual-Functional PLLBased Polymer. To better evaluate the contribution of each functional component of PLL-mal(26\%)-PNA-CEEEEE to the antifouling properties of the surface layer, we verified the antifouling behavior of layers, including the functional components with a different combination than in PLL$\operatorname{mal}(26 \%)$-PNA-CEEEEE. We tested the antifouling properties of such layers by measuring $\% R$ changes produced by the running buffer (PBS) after the exposure of the surface to diluted pooled human plasma (10\% in PBS, $30 \mathrm{~min}$ ). Plasma components nonspecifically adsorbed onto the surface are responsible for the detected SPRI signal change. For this reason, we calculated the mass of adsorbate per unit area $(\Gamma)$ from the measured $\Delta \% R$ value.

Figure 4 shows representative SPRI kinetic profiles detected for the exposure of (a) PLL/PNA/CEEEEE (no maleimide groups in PLL), (b) PNA-mal(26\%) (no PNA, no CEEEEE), (c) PLL-mal(26\%)-PNA (no CEEEEE), and (d) PLLmal(26\%)-PNA-CEEEEE, to $10 \%$ plasma in PBS. To test the antifouling polymers, we used the PNA-WT probe. SPRI data for the other investigated layers are reported in the Supporting Information (Figures S8, S10, and S12), whereas SPRI data referring to the surface immobilization of various functional components are reported in Figure S9.
The adsorption of proteins from plasma samples on some of the investigated layers (i.e., PLL-mal(26\%)-PNA, PLLmal(26\%)-PNA-CEEEEE, and PLL/PNA; Figures $4 \mathrm{~b}-\mathrm{d}$ and S8) generates an initial SPR signal increase followed by a slowly decreasing kinetic profile. The peculiar profile is the likely consequence of the electrostatic interaction between charged plasma proteins and the charged PLL polymers bearing different amounts of neutral (PNA) and charged (PLL and CEEEEE) species. Similar kinetic profiles have also been reported for the adsorption of plasma proteins on other charged polymers. ${ }^{57,58}$

We tested first PLL-based layers bearing no maleimide functionalities (Figures $4 \mathrm{a}$ and S8) to evaluate the role played by maleimide groups in driving the assembly of layers showing enhanced antifouling capacity. Thiol moieties of PNA and CEEEEE structures directly interact with the gold surface of the SPRI sensor (Figure S10). PNA and CEEEEE also may interact nonspecifically with PLL, being the electrostatic interaction between PLL and CEEEEE the primary driving force involved. Such interactions compete with and complement the reaction between thiol moieties and maleimide groups of PLL-mal(26\%), causing a nonsignificant change in the number of PNA and CEEEEE molecules adsorbed on sensor surfaces modified with PLL and PLL-mal(26\%), respectively. An evident change in the SPRI kinetic profile for CEEEEE adsorption accounting for a transition from a diffusion-limited process to a reaction kinetic-controlled process was observed when maleimide units were present on the surface (Figure S11). The fouling resistance of the surface layers we tested can be assessed through the value of $\Gamma$ calculated from the SPRI experiments performed with human plasma (Table 2).

All PLL-based layers we tested exhibited some fouling resistance. The adsorption of the neutral PNA probes on cationic PLL produced no significant variation in the fouling resistance of the surface ( $\Gamma$ changed from $535 \pm 17 \mathrm{ng} \mathrm{cm}^{-2}$ to $\left.558 \pm 54 \mathrm{ng} \mathrm{cm}^{-2}\right)$. The lowest $\Gamma$ value $(\Gamma=347 \pm 45 \mathrm{ng}$ 
$\mathrm{cm}^{-2}$ ) we measured for PLL-based layers bearing no maleimide groups, corresponding to the best fouling resistance, refers to the layer obtained by adsorbing the anionic CEEEEE peptide on PLL/PNA (Table 2 and Figure 4a). Such a result confirmed the critical role of charges on the surface layer's fouling resistance.

We then tested the fouling resistance of PLL-mal(26\%)based layers bearing maleimide groups. Such layers showed better fouling resistance than the corresponding layers bearing no maleimide units (Table 2). The better fouling resistance of PLL-mal (26\%) compared to PLL $(\Gamma=364 \pm 13$ vs $535 \pm 17$ $\mathrm{ng} \mathrm{cm}{ }^{-2}$ ) is attributed to the oligo(ethylene glycol) structure of the arm that we covalently added to PLL to introduce maleimide units. As already observed for PLL, the introduction of neutral PNA produced no significant changes in the fouling resistance $\left(\Gamma=381 \pm 31 \mathrm{ng} \mathrm{cm}{ }^{-2}\right)$ of the resulting surface.

The adsorption of CEEEEE on PLL-mal(26\%) significantly improved the fouling resistance of the surface as already observed for PLL. In particular, PLL-mal(26\%)-CEEEEE provided the best fouling resistance among the tested systems with a remarkable reduction of $\left.\Gamma(48 \mathrm{ng} \mathrm{cm})^{-2}\right),{ }^{32}$ which testifies the reduction of the total amount of plasma components adsorbed on PLL-mal(26\%)-CEEEEE. The coupling reaction between CEEEEE and the maleimide moiety creates a charge-balanced system that enhances the antifouling capacity of the PLL-mal(26\%)-based layer. ${ }^{32}$ At physiological $\mathrm{pH}$, plasma proteins can interact via electrostatic forces with cationic polymers such as PLL and PLL-mal(26\%), by raising the mass of adsorbate per unit area (Table 2 and Figure $4 a-c$ ). CEEEEE anionic side chains covalently attached to PLL through maleimide groups shield positive charges, thereby reducing the electrostatic attraction of the plasma proteins on the surface (Table 2 and Figure 4d). ${ }^{32}$

The sequential adsorption of PNA and CEEEEE (Figure 3) did not alter the fouling resistance of the PLL-mal(26\%)CEEEEE charge-balanced surface $(\Gamma=46 \pm 34$ vs $48 \mathrm{ng}$ $\mathrm{cm}^{-232}$ ), thus offering the opportunity to fabricate a surface layer combining the enhanced fouling resistance with the capacity of PNA probes to hybridize the complementary sequences of DNA in plasma samples specifically.

We tested the fouling resistance of the surface obtained by adsorbing the anionic peptide EEEEE bearing no thiol moiety on PLL-mal(26\%) to confirm the synergistic contribution to the antifouling properties of both the covalent coupling between the thiolated CEEEEE and maleimide groups and the repulsive electrostatic interaction between plasma components and the mixed-charge PLL-mal(26\%)-based layer. We also detected SPRI curves during the exposure of (a) PLL-mal(26\%)/EEEEE and (b) PLL-mal(26\%)-CEEEEE surfaces to plasma (10\% in PBS) (Figure S12). The two layers provided different $\Delta \% R$ changes both during the adsorption of the diluted human plasma and when the PBS baseline was established after the plasma adsorption. PLL-mal(26\%)/ $\operatorname{EEEEE}\left(\Gamma=213 \pm 53 \mathrm{ng} \mathrm{\textrm {cm } ^ { - 2 }}\right)$ did not improve the antifouling property of PLL-mal(26\%)-CEEEEE, thus confirming the requirement for the interaction between the thiol group of the anionic peptide and maleimide groups introduced in the PLL structure.

Nanoparticle-Enhanced SPRI Detection of Tumor DNA in Human Plasma Using the Dual-Functional PLL-Based Polymer. Nanostructure-enhanced SPR has been already shown to allow detection of nucleic acids $^{15,54,59,60}$ including microRNAs, ${ }^{61}$ protein biomarkers, ${ }^{62,63}$ and small molecules ${ }^{64}$ with attomolar sensitivity. ${ }^{65}$ The ultrahigh sensitivity achieved with metallic nanoparticles has been attributed to dielectric constant enhancement from nanoparticles clustered on the SPR chip due to processes such as magnetic interaction ${ }^{66}$ or nucleic acid sequence-induced aggregation. ${ }^{67}$ Possibilities offered by ultrasensitive detection methods based on nanoparticle-enhanced SPR biosensors have been recently reviewed. ${ }^{68}$ Challenges and solutions for ultrasensitive biosensing have also been discussed with a broader perspective. ${ }^{69}$ Here, we demonstrate that the nanoparticle-enhanced SPRI ultrasensitive detection can be implemented for the direct detection of ctDNA in human plasma.

To demonstrate that the PLL-mal(26\%)-PNA-CEEEEE surface layer combined with nanoparticle-enhanced SPRI detection of mutated and wild-type DNAs in human plasma provides a new platform for the direct analysis of plasma samples from cancer patients, we adsorbed PLL-mal(26\%) on the SPRI gold surface and immobilized PNA probes $(0.1 \mu \mathrm{M}$ in PBS, flow rate $10 \mu \mathrm{L} \mathrm{min}{ }^{-1}$ ) as already described (Figures 3 and 1a). We obtained the spatial separation of the immobilized PNA probes complementary to wild-type and p.G12D-mutated DNA target sequences using a microfluidic device bearing parallel microchannels (Figure 1). We then introduced CEEEEE (surface density $21 \times 10^{12}$ molecules $\mathrm{cm}^{-2}$ ) to obtain the dual-functional layer.

We analyzed plasma samples (10\% in PBS) spiked with p.G12D-mutated or wild-type gDNAs at a final concentration of $5 \mathrm{pg} \mu \mathrm{L}^{-1}\left(\sim 2.5 \mathrm{aM}\right.$ assuming $\left.\mathrm{MW}=1.9 \times 10^{12}\right)$ as a proxy of ctDNA detection in plasma from cancer patients and cfDNA detection in plasma from a healthy donor, respectively.

The spiked samples were introduced in the microfluidic device for adsorption on PNA probes (Figure 1b) after sonication $(3 \mathrm{~min})$, vortexing $(1 \mathrm{~min})$, heating at $95{ }^{\circ} \mathrm{C}(5$ $\mathrm{min}$ ), and incubation for $1 \mathrm{~min}$ on ice before the analysis.

SPRI responses detected after the adsorption of plasma


gDNA on PLL-mal(26\%)-PNA-CEEEEE layers bearing PNAWT and PNA-G12D (Figure S13) confirmed the antifouling properties of the surface layer and provided no helpful signal to identify the specific interactions between p.G12D-mutated gDNA and the complementary PNA-G12D probe. After evaluating SPRI responses detected for the adsorption of plasma spiked with wild-type gDNA (Figure S13), we have drawn the same conclusions.

As the last step of the assay, we used functionalized AuNPs to enhance plasmonic detection and produce SPRI signals useful to detect p.G12D-mutated and wild-type gDNAs in plasma samples selectively (Figure 1c). We obtained AuNP@ KRAS by conjugating AuNPs with an oligonucleotide complementary to an exposed DNA region of the KRAS exon carrying the p.G12D mutation or the corresponding wildtype sequence not involved in the hybridization with PNA-WT or PNA-G1D probes. ${ }^{15,70}$ We obtained the SPRI signal enhancement by adsorbing AuNP@KRAS dispersion (0.1

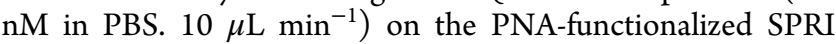
surface after the already mentioned plasma adsorption and a 10 min washing step with PBS.

After AuNP@KRAS enhancement, the SPRI assay distinguished plasma samples carrying wild-type or p.G12D-mutated gDNAs $\left(5 \mathrm{pg} \mu \mathrm{L}^{-1}\right)$. Nanoparticle enhancement highlighted the preferential interaction of wild-type DNA with PNA-WT (Figure 5a), whereas p.G12D-mutated DNA preferentially 
interacted with the PNA-G12D probe (Figure $5 \mathrm{~b}$ ), as expected.


Figure 5. Representative time-dependent SPRI curves for the adsorption of AuNP@KRAS on (a) wild-type and (b) p.G12Dmutated gDNA in plasma previously adsorbed on surface-immobilized PNA-WT and PNA-G12D probes.

In particular, the ratio of $\Delta \% R$ values detected after the adsorption of AuNP@KRAS on surfaces resulting from the interaction of the selected plasma sample with PLL-mal(26\%)PNA-CEEEEE surface layers modified with PNA-WT and PNA-G12D provides better visualization of the discrimination between plasma samples with wild-type or p.G12D-mutated DNA.

The shape of SPR profiles detected for AuNP@KRAS adsorption (Figure 5) is the consequence of diffusion-limited kinetics $^{71}$ arising because the rate of AuNP@KRAS nanoparticle diffusion to the surface is slower than the kinetics of the interaction between oligonucleotides immobilized on AuNP@KRAS and DNA target molecules captured by PNA probes. Figure 5 highlights the difference in SPR signals due to the single base mismatch detection. The detection of WT or p.G12D single base mutated DNAs causes an inversion of the order of curves shown in Figure $5 a$ and $5 b$, respectively. Nonspecifically adsorbed DNA fragments can trigger nanoparticle aggregation on the surface due to the alteration of the local charge balance they cause, as discussed elsewhere. ${ }^{67}$ Considering the possible contribution of the nonspecific AuNP@KRAS adsorption on the detected SPR signal, we calculated the ratio between SPR signals detected from the two surfaces differing from each other only for the immobilized PNA probe (PNA-WT and PNA-G12D), which differ by only one base. PNA selectivity and cross-reactivity in the PBS buffer have been already tested for similar systems, as reported elsewhere. ${ }^{15}$ The use of PNA clamp sequences and the finetuning of the assay temperature may be evaluated to improve the discrimination between AuNP@KRAS enhanced signals.

Figure 6 summarizes results from replicated independent analyses of plasma samples spiked with wild-type or p.G12D-

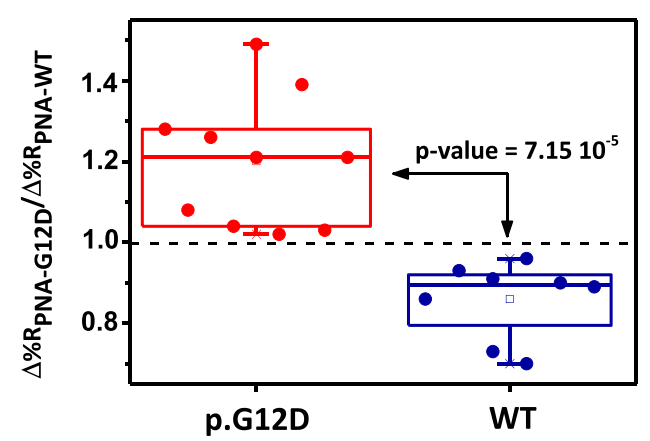

Figure 6. $\Delta \% R_{\mathrm{PNA}-\mathrm{G} 12 \mathrm{D}} / \Delta \% R_{\mathrm{PNA}-\mathrm{WT}}$ ratio values obtained from replicated experiments aimed at detecting wild-type (WT) and p.G12D gDNAs in $10 \%$ diluted human plasma samples $\left(5 \mathrm{pg} \mu \mathrm{L}^{-1}\right)$. Ratios were obtained by considering $\Delta \% R$ values after $1000 \mathrm{~s}$ of adsorption of AuNP@KRAS. The ratio considers SPRI responses $(\Delta \% R)$ referred to PNA-G12D $\left(\Delta \% R_{\mathrm{PNA}-\mathrm{G} 12 \mathrm{D}}\right)$ and PNA-WT $(\Delta \%$ $R_{\mathrm{PNA}-\mathrm{WT}}$ ) probes when the same plasma sample was detected. Wildtype $\left(\Delta \% R_{\mathrm{PNA}-\mathrm{G} 12 \mathrm{D}} / \Delta \% R_{\mathrm{PNA}-\mathrm{WT}}\right.$ ratio population mean confidence interval $(\mathrm{CI})$ at the $95 \%$ level $=0.86 \pm 0.09$, replicate measurements $n$ $=8)$ and p.G12D $\left(\Delta \% R_{\mathrm{PNA}-\mathrm{G} 12 \mathrm{D}} / \Delta \% R_{\mathrm{PNA}-\mathrm{WT}}\right.$ ratio population mean $\mathrm{CI}=1.20 \pm 0.16$, replicate measurements $n=10)$ samples generated significantly different $\Delta \% R_{\mathrm{PNA}-\mathrm{G} 12 \mathrm{D}} / \Delta \% R_{\mathrm{PNA}-\mathrm{WT}}$ ratios $(t$-test, level $95 \%$, two-tailed, $p$-value $\left.=7.15 \times 10^{-5}\right)$. A dotted line is shown to highlight better the values of the $\Delta \% R_{\mathrm{PNA}-\mathrm{G} 12 \mathrm{D}} / \Delta \% R_{\mathrm{PNA}-\mathrm{WT}}$ ratio below and beyond 1 .

mutated gDNAs $\left(5 \mathrm{pg} \mu \mathrm{L}^{-1}\right)$. We performed the experiments analyzing in parallel wild-type and p.G12D-mutated samples.

The $\Delta \% R_{\text {PNA-G12D }} / \Delta \% R_{\text {PNA-WT }}$ ratio for p.G12D-mutated DNA (population mean confidence interval at the 95\% level for the ratio $\mathrm{CI}=1.20 \pm 0.16$, replicate analyses $n=10$ ) was significantly different from that for wild-type DNA $(\Delta \%$ $R_{\text {PNA-G12D }} / \Delta \% R_{\text {PNA-WT }} 95 \% \mathrm{CI}=0.86 \pm 0.09, n=8$; two-tailed $t$-test, level 95\%, $p$-value $\left.=7.15 \times 10^{-5}\right)$.

To evaluate the assay performance with a single-donor plasma (sample \#4) instead of pooled plasma samples, we generated a calibration curve (Figure $7 \mathrm{a}$ ) using plasma from an individual healthy donor. In particular, we investigated a dynamic range $\left(0.5-20.0 \mathrm{pg} \mu \mathrm{L}^{-1}\right)$ for p.G12D-mutated DNA in plasma with typical concentrations of ctDNA in liquid biopsy from cancer patients. ${ }^{72}$ The detection of higher ctDNA concentrations involves tuning different parameters, such as nanoparticle concentration and PNA probe surface density, which falls outside of the scope of this paper. We estimated the detection limit of the assay using the four-parameter logistic regression procedure described elsewhere. ${ }^{73,74}$ We used the following four-parameter logistic equation for the experimental data fitting $y=d+(a-d) /(1+([\mathrm{p} . \mathrm{G} 12 \mathrm{D}]+2 / c) \wedge b)$, where $\mathrm{y}$ corresponds to $\Delta \% R_{\mathrm{PNA}-\mathrm{G} 12 \mathrm{D}} / \Delta \% R_{\mathrm{PNA}-\mathrm{WT}}$ values at the spiked p.G12D concentration [p. G12D] in plasma samples. Figure $7 \mathrm{~b}$ shows the four-parameter logistic fit (gray line, adj. $\left.R^{2}=0.990\right)$ with lower and upper $95 \%$ prediction limits (dashed gray lines). We obtained both the minimum detectable concentration $\left(\mathrm{MDC}=0.58 \mathrm{pg} \mu \mathrm{L}^{-1}\right)$ and the 

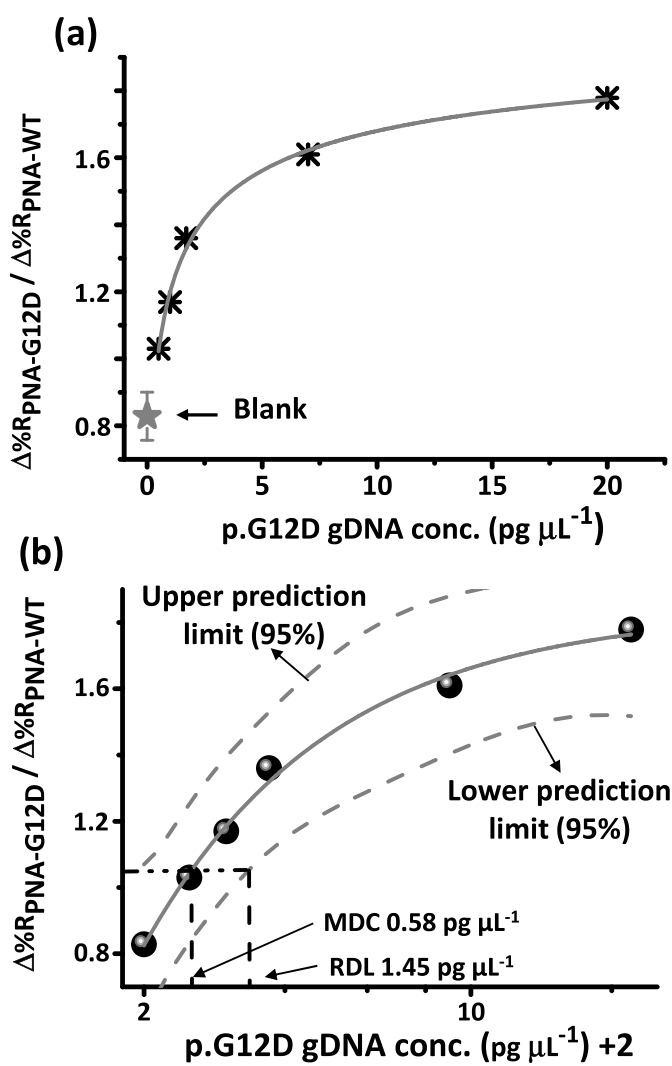

Figure 7. (a) SPRI calibration curve of different p.G12D-mutated gDNA concentrations spiked in $10 \%$ diluted plasma of an individual healthy donor (sample \#4). The average $\Delta \% R$ ratio generated by blank samples is shown (gray star, $n=5$ ). (b) Four-parameter logistic function for experimental data fitting of the SPRI calibration curve is shown (gray line). p.G12D gDNA concentration values are reported on a log-scale axis. We added 2 to the actual concentration to include the negative control (p.G12D concentration $=0$ ) in the fitting procedure, as described in ref 73 . The same number was subtracted after the end of the process. The best fit $\left(\operatorname{adj} . R^{2}=0.987\right)$ was obtained using the equation $y=d+(a-d) /(1+([$ p.G12D $]+2 /$ c) $\wedge b)$ with the following parameters: $a=-51.3582 ; b=1.10261 ; c=$ 0.04994; $d=1.83694$. By the data fitting, the minimum detectable concentration $\left(\mathrm{MDC}=0.58 \mathrm{pg} \mu \mathrm{L}^{-1}\right)$ and the reliable detection limit $\left(\mathrm{RDL}=1.45 \mathrm{pg} \mu \mathrm{L}^{-1}\right)$ were estimated as DNA concentrations corresponding to the interpolated intersections of the lower asymptote of the upper $95 \%$ prediction limit with the four-parameter logistic fit curve and the lower $95 \%$ prediction limit, respectively.

reliable detection limit $\left(\mathrm{RDL}=1.45 \mathrm{pg} \mu \mathrm{L}^{-1}\right)$ as DNA concentrations corresponding to the interpolated intersections of the lower asymptote of the upper $95 \%$ prediction limit with the four-parameter logistic fit curve and the lower 95\% prediction limit, respectively. The estimated MDC and RDL parameters provide an improved analytical sensitivity compared to other plasmonic platforms recently described for circulating biomarker detection in biofluids. ${ }^{75}$

In light of the promising results obtained from experiments with spiked plasma samples, we tested the assay performance for the direct detection of KRAS-mutated ctDNA in plasma of CRC patients as a real-world application of liquid biopsy for cancer diagnosis. With this aim, we analyzed sample pt\#34 (CRC patient) with p.G13D KRAS-mutated ctDNA and sample \#4 from an individual healthy donor. Figure 8 shows the results $\left(\Delta \% R_{\mathrm{PNA}-\mathrm{G} 13 \mathrm{D}} / \Delta \% R_{\mathrm{PNA}-\mathrm{WT}}\right)$ obtained from replicated analyses of liquid biopsies.

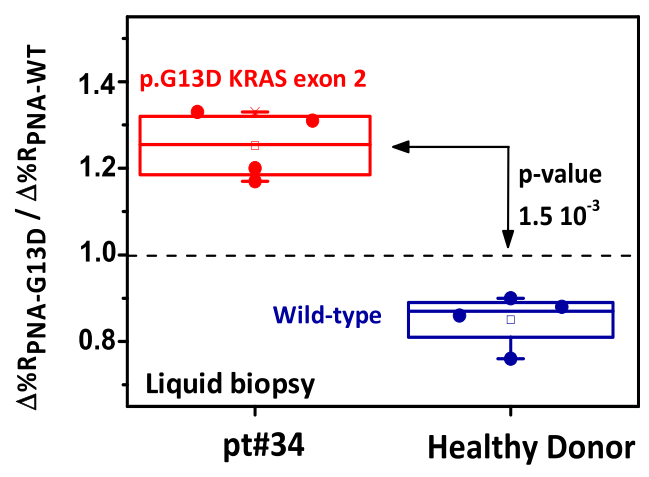

Figure 8. Box plot of the $\Delta \% R_{\mathrm{PNA}-\mathrm{G} 13 \mathrm{D}} / \Delta \% R_{\mathrm{PNA}-\mathrm{WT}}$ ratio calculated after the adsorption of AuNP@KRAS. Plasma samples from the CRC patient with p.G13D KRAS-mutated ctDNA (sample pt\#34) provided values greater than 1 , whereas samples from the healthy donor (sample \#4, wild-type cfDNA) provided significantly different $\Delta \%$ $R_{\mathrm{PNA}-\mathrm{G} 13 \mathrm{D}} / \Delta \% R_{\mathrm{PNA}-\mathrm{WT}}$ ratios $(t$-test, level $95 \%$, two-tailed, $p$-value $=$ $\left.1.5 \times 10^{-3}\right)$.

The $\Delta \% R_{\text {PNA-G13D }} / \Delta \% R_{\text {PNA-WT }}$ ratio for p.G13D-mutated ctDNA (population mean confidence interval at the 95\% level for the ratio $\mathrm{CI}=1.25 \pm 0.08$, replicate analyses $n=4$ ) was significantly different from that detected for cfDNA from a healthy donor $\left(\Delta \% R_{\text {PNA-G13D }} / \Delta \% R_{\text {PNA-WT }} 95 \% \mathrm{CI}=0.85 \pm\right.$ $0.06, n=4$; two-tailed $t$-test, level 95\%, $p$-value $\left.=1.5 \times 10^{-3}\right)$.

These data confirm that the designed nanoparticle-enhanced SPRI assay combined with the dual-functional PLL surface layer provides a new platform for the straightforward detection of tumor DNA in plasma samples. The new system introduces significant improvements as compared to the state-of-the-art technologies for liquid biopsy analysis.

The preanalytical workflow of a typical liquid biopsy test envisages DNA isolation from blood and then analysis by either NGS or dPCR. This includes a typical double-spin whole blood/plasma protocol (about $30 \mathrm{~min}$ ), the purification on an affinity matrix under negative pressure/magnetic field and elution $(75 \mathrm{~min})$, and final DNA quantification $(5 \mathrm{~min}){ }^{23}$ The subsequent analytical phase lasts from 4 to $18 \mathrm{~h}$ ( $\mathrm{dPCR}$ and NGS, respectively), including the data elaboration time, and this time cannot be compressed because both dPCR and NGS are endpoint methods. ${ }^{24,25}$ In contrast, the new platform significantly cuts down wet-lab time $(10 \mathrm{~min})$ and provides a real-time readout. Most importantly, it avoids several cumbersome steps, e.g., temperature cycling, sample carryover, sample transfer to different pieces of equipment such as different types of centrifuges (a wide range of $\mathrm{g}$ forces is normally needed), purification devices, fluorimeter, and test tubes for volume/concentration adjustments. ${ }^{18}$ Altogether, the total preanalytical turnaround time is 115 vs $10 \mathrm{~min}$ for conventional testing and dual-functional PLL SPRI, respectively. The analytical time is $4-18$ vs $2 \mathrm{~h}$. The total turnaround time is $350 \mathrm{~min}$ or $48 \mathrm{vs} 2.5 \mathrm{~h}$. The hands-on time is rather similar, but the new SPRI assay condensates all steps within a narrow time-lapse, whereas NGS requires at least an overnight step and the person-time intensive use hands-off time is fractionated and poorly usable.

The new platform also introduces substantial improvements compared to the recently introduced nanoparticle-enhanced SPRI assay for detecting ctDNA in plasma samples. ${ }^{15}$ The preanalytical processing of the plasma sample no longer involves the $1.5 \mathrm{~h}$ lasting treatment of plasma samples with proteinase $\mathrm{K}$, and the SPRI sensor surface is not treated with 
dithiothreitol or other blocking additives after the plasma adsorption as a consequence of the surface fouling resistance introduced by PLL-mal(26\%)-PNA-CEEEEE.

\section{CONCLUSIONS}

This paper demonstrates that the combined use of the new PLL-mal(26\%)-PNA-CEEEEE surface layer and nanoparticleenhanced SPRI provides a platform for the straightforward detection of tumor DNA in the plasma of cancer patients. In particular, the key achievement described herein is the design of a new dual-functional low-fouling PLL-based polymer, containing both anionic oligopeptide and neutral PNA probe side chains. We show that this very focused technical improvement has a considerable implication because it virtually eliminates sample pretreatment from patient serum samples' processing. In other words, blood may be processed for liquid biopsy exactly as for routine blood biochemistry, virtually eliminating dedicated liquid biopsy pipelines, and contributing to fit liquid biopsy into the standard hematological routine. The densely immobilized CEEEEE peptides and the cationic PLL-based structure create a mixed-charge layer that inhibits the unspecific adsorption of components of complex matrices such as the human plasma. At the same time, the sparsely attached PNA probes provide the genomic target binding partners. We have demonstrated that components of the dual-functional PLL polymer perform a synergistic antifouling effect. We have studied the role played by each component of the antifouling surface layer by providing information that may be useful for the development of different biosensing platforms. Nanoparticle-enhanced SPRI sandwich assays using the new dual-functional surface layer enable the specific detection of tumor KRAS single-point mutated gDNA, at the attomolar level and directly in human plasma, and ctDNA in a liquid biopsy sample from a CRC patient with no need for preliminary ctDNA isolation, purification, and amplification. Thus, the new platform is not subject to several constraints affecting methods currently applied in the clinical practice for cancer diagnosis, which, instead, require both complicated and time-consuming sample processing and PCR amplification of ctDNA. PLL-mal(26\%)PNA-CEEEEE antifouling properties also simplify the nanoparticle-enhanced SPRI assays compared to a recently introduced NESPRI-based approach.

In conclusion, the SPRI biosensing strategy based on PLLmal(26\%)-PNA-CEEEEE offers a rapid, amplification-free, and straightforward detection of tumor-derived materials circulating in biological fluids and makes a significant contribution to the improvement of early clinical diagnosis and personalized medicine in liquid biopsy. Hopefully, biosensors with lowfouling surfaces will contribute a much-needed technical solution and will make liquid biopsy a truly routine assay, widely available, and fully integrated into advanced health-care systems.

\section{ASSOCIATED CONTENT}

\section{s) Supporting Information}

The Supporting Information is available free of charge at https://pubs.acs.org/doi/10.1021/acssensors.1c00360.

Characterization of oligopeptide; PNA probes and gold nanoparticles; and SPRI data (PDF)

\section{AUTHOR INFORMATION}

\section{Corresponding Authors}

Jurriaan Huskens - Department of Molecules \& Materials, MESA+ Institute for Nanotechnology, Faculty of Science \& Technology, University of Twente, 7500 AE Enschede, The Netherlands; (1) orcid.org/0000-0002-4596-9179;

Email: j.huskens@utwente.nl

Giuseppe Spoto - Department of Chemical Sciences, University of Catania, 95122 Catania, Italy; INBB, Istituto Nazionale di Biostrutture e Biosistemi, 00136 Roma, Italy; (1) orcid.org/0000-0003-3201-8689;

Email: giuseppe.spoto@unict.it

\section{Authors}

Noemi Bellassai - Department of Chemical Sciences, University of Catania, 95122 Catania, Italy

Roberta D'Agata - Department of Chemical Sciences, University of Catania, 95122 Catania, Italy; INBB, Istituto Nazionale di Biostrutture e Biosistemi, 00136 Roma, Italy

Almudena Marti - Department of Molecules \& Materials, MESA+ Institute for Nanotechnology, Faculty of Science \& Technology, University of Twente, 7500 AE Enschede, The Netherlands

Andrea Rozzi - Department of Chemistry, Life Sciences and Environmental Sustainability, University of Parma, 43124 Parma, Italy

Stefano Volpi - Department of Chemistry, Life Sciences and Environmental Sustainability, University of Parma, 43124 Parma, Italy

Matteo Allegretti - Oncogenomics and Epigenetics, IRCCS Regina Elena National Cancer Institute, 00144 Rome, Italy

Roberto Corradini - Department of Chemistry, Life Sciences and Environmental Sustainability, University of Parma, 43124 Parma, Italy; INBB, Istituto Nazionale di Biostrutture e Biosistemi, 00136 Roma, Italy; 이이.org/ 0000-0002-8026-0923

Patrizio Giacomini - Oncogenomics and Epigenetics, IRCCS Regina Elena National Cancer Institute, 00144 Rome, Italy; (1) orcid.org/0000-0001-6109-1709

Complete contact information is available at:

https://pubs.acs.org/10.1021/acssensors.1c00360

\section{Author Contributions}

The manuscript was written through contributions of all authors. All authors have approved the final version of the manuscript.

\section{Notes}

The authors declare no competing financial interest.

\section{ACKNOWLEDGMENTS}

This work was supported by European Union's Horizon 2020 research and innovation program under grant agreement no. 633937, project ULTRAPLACAD. A.M. and J.H. acknowledge financial support from the MESA+Institute for Nanotechnology, University of Twente. The present work has also benefited from the equipment and framework of the COMP-HUB Initiative, funded by the "Departments of Excellence" program of the Italian Ministry for Education, University and Research (MIUR, 2018-2022) for the Department of Chemistry, Life Sciences and Environmental Sustainability of the University of Parma. M.A. is a recipient of an AIRC 3 year fellowship (id. 19504). 


\section{REFERENCES}

(1) Heitzer, E.; Haque, I. S.; Roberts, C. E. S.; Speicher, M. R. Current and Future Perspectives of Liquid Biopsies in GenomicsDriven Oncology. Nat. Rev. Genet. 2019, 20, 71-88.

(2) Michael, E. Taking Cancer out of Circulation. Nature 2020, 579, S6-S8.

(3) Palmirotta, R.; Lovero, D.; Cafforio, P.; Felici, C.; Mannavola, F.; Pellè, E.; Quaresmini, D.; Tucci, M.; Silvestris, F. Liquid Biopsy of Cancer: A Multimodal Diagnostic Tool in Clinical Oncology. Ther. Adv. Med. Oncol. 2018, 10, No. 1758835918794630.

(4) De Rubis, G.; Rajeev Krishnan, S.; Bebawy, M. Liquid Biopsies in Cancer Diagnosis, Monitoring, and Prognosis. Trends Pharmacol. Sci. 2019, 40, 172-186.

(5) Merker, J. D.; Oxnard, G. R.; Compton, C.; Diehn, M.; Hurley, P.; Lazar, A. J.; Lindeman, N.; Lockwood, C. M.; Rai, A. J.; Schilsky, R. L.; Tsimberidou, A. M.; Vasalos, P.; Billman, B. L.; Oliver, T. K.; Bruinooge, S. S.; Hayes, D. F.; Turner, N. C. Circulating Tumor DNA Analysis in Patients With Cancer: American Society of Clinical Oncology and College of American Pathologists Joint Review. Arch. Pathol. Lab. Med. 2018, 142, 1242-1253.

(6) Peters, D. L.; Pretorius, P. J. Origin, Translocation and Destination of Extracellular Occurring DNA - A New Paradigm in Genetic Behaviour. Clin. Chim. Acta 2011, 412, 806-811.

(7) Jen, J.; Wu, L.; Sidransky, D. An Overview on the Isolation and Analysis of Circulating Tumor DNA in Plasma and Serum. Ann. N. Y. Acad. Sci. 2000, 906, 8-12.

(8) Volckmar, A. L.; Sültmann, H.; Riediger, A.; Fioretos, T.; Schirmacher, P.; Endris, V.; Stenzinger, A.; Dietz, S. A Field Guide for Cancer Diagnostics Using Cell-Free DNA: From Principles to Practice and Clinical Applications and the Subpopulation of CtDNA. Genes, Chromosomes Cancer 2018, 57, 123-139.

(9) Zhang, Y.; Noji, H. Digital Bioassays: Theory, Applications, and Perspectives. Anal. Chem. 2017, 89, 92-101.

(10) Das, J.; Ivanov, I.; Montermini, L.; Rak, J.; Sargent, E. H.; Kelley, S. O. An Electrochemical Clamp Assay for Direct, Rapid Analysis of Circulating Nucleic Acids in Serum. Nat. Chem. 2015, 7, 569-575.

(11) Chen, D.; Wu, Y.; Hoque, S.; Tilley, R. D.; Gooding, J. J. Rapid and Ultrasensitive Electrochemical Detection of Circulating Tumor DNA by Hybridization on the Network of Gold-Coated Magnetic Nanoparticles. Chem. Sci. 2021, 12, 5196-5201.

(12) Soda, N.; Rehm, B. H. A.; Sonar, P.; Nguyen, N. T.; Shiddiky, M. J. A. Advanced Liquid Biopsy Technologies for Circulating Biomarker Detection. J. Mater. Chem. B 2019, 7, 6670-6704.

(13) Thompson, M.; Blaszykowski, C.; Sheikh, S.; RodriguezEmmenegger, C.; de los Santos Pereira, A. Biological Fluid-Surface Interactions in Detection and Medical Devices, Detection Science; Royal Society of Chemistry: Cambridge, 2016.

(14) Liu, B.; Liu, X.; Shi, S.; Huang, R.; Su, R.; Qi, W.; He, Z. Design and Mechanisms of Antifouling Materials for Surface Plasmon Resonance Sensors. Acta Biomater. 2016, 40, 100-118.

(15) D’Agata, R.; Bellassai, N.; Allegretti, M.; Rozzi, A.; Korom, S.; Manicardi, A.; Melucci, E.; Pescarmona, E.; Corradini, R.; Giacomini, P.; Spoto, G. Direct Plasmonic Detection of Circulating RAS Mutated DNA in Colorectal Cancer Patients. Biosens. Bioelectron. 2020, 170, No. 112648.

(16) Devonshire, A. S.; Whale, A. S.; Gutteridge, A.; Jones, G.; Cowen, S.; Foy, C. A.; Huggett, J. F. Towards Standardization of CellFree DNA Measurement in Plasma: Controls for Extraction Efficiency, Fragment Size Bias and Quantification. Anal. Bioanal. Chem. 2014, 406, 6499-6512.

(17) Sorber, L.; Zwaenepoel, K.; Deschoolmeester, V.; Roeyen, G.; Lardon, F.; Rolfo, C.; Pauwels, P. A Comparison of Cell-Free DNA Isolation Kits: Isolation and Quantification of Cell-Free DNA in Plasma. J. Mol. Diagn. 2017, 19, 162-168.

(18) Ignatiadis, M.; Sledge, G. W.; Jeffrey, S. S. Liquid Biopsy Enters the Clinic - Implementation Issues and Future Challenges. Nat. Rev. Clin. Oncol. 2021, 18, 297-312.
(19) Gerber, T.; Taschner-Mandl, S.; Saloberger-Sindhöringer, L.; Popitsch, N.; Heitzer, E.; Witt, V.; Geyeregger, R.; Hutter, C.; Schwentner, R.; Ambros, I. M.; Ambros, P. F. Assessment of PreAnalytical Sample Handling Conditions for Comprehensive Liquid Biopsy Analysis. J. Mol. Diagn. 2020, 22, 1070-1086.

(20) Johansson, G.; Andersson, D.; Filges, S.; Li, J.; Muth, A.; Godfrey, T. E.; Ståhlberg, A. Considerations and Quality Controls When Analyzing Cell-Free Tumor DNA. Biomol. Detect. Quantif. 2019, 17, No. 100078.

(21) Geeurickx, E.; Hendrix, A. Targets, Pitfalls and Reference Materials for Liquid Biopsy Tests in Cancer Diagnostics. Mol. Aspects Med. 2020, 72, No. 100828.

(22) Li, Z.; Yi, L.; Gao, P.; Zhang, R.; Li, J. The Cornerstone of Integrating Circulating Tumor DNA into Cancer Management. Biochim. Biophys. Acta, Rev. Cancer 2019, 1871, 1-11.

(23) QiagenQIAamp Circulating Nucleic Acid Handbook, Qiagen, 2019.

(24) Guide, U. QuantStudio 3D Digital PCR System Life Technol. 2013, p 34.

(25) Thermo Fisher Scientific, Oncomine Pan-Cancer Cell-Free Assay User Guide (Pub. No. MAN0017064 C). 2018, pp 12-14.

(26) Vaisocherová, H.; Brynda, E.; Homola, J. Functionalizable LowFouling Coatings for Label-Free Biosensing in Complex Biological Media: Advances and Applications. Anal. Bioanal. Chem. 2015, 407, 3927-3953.

(27) Nir, S.; Reches, M. Bio-Inspired Antifouling Approaches: The Quest towards Non-Toxic and Non-Biocidal Materials. Curr. Opin. Biotechnol. 2016, 39, 48-55.

(28) Damodaran, V. B.; Murthy, N. S. Bio-Inspired Strategies for Designing Antifouling Biomaterials. Biomater. Res. 2016, 20, No. 18.

(29) Lowe, S.; O’Brien-Simpson, N. M.; Connal, L. A. Antibiofouling Polymer Interfaces: Poly(Ethylene Glycol) and Other Promising Candidates. Polym. Chem. 2015, 6, 198-212.

(30) Cui, M.; Wang, Y.; Jiao, M.; Jayachandran, S.; Wu, Y.; Fan, X.; Luo, X. Mixed Self-Assembled Aptamer and Newly Designed Zwitterionic Peptide as Antifouling Biosensing Interface for Electrochemical Detection of Alpha-Fetoprotein. ACS Sens. 2017, 2, 490494.

(31) Zhang, Y.; Liu, Y.; Ren, B.; Zhang, D.; Xie, S.; Chang, Y.; Yang, J.; Wu, J.; Xu, L.; Zheng, J. Fundamentals and Applications of Zwitterionic Antifouling Polymers. J. Phys. D: Appl. Phys. 2019, 52, No. 403001.

(32) Bellassai, N.; Marti, A.; Spoto, G.; Huskens, J. Low-Fouling, Mixed-Charge Poly-l-Lysine Polymers with Anionic Oligopeptide Side-Chains. J. Mater. Chem. B 2018, 6, 7662-7673.

(33) Shen, J.; Du, M.; Wu, Z.; Song, Y.; Zheng, Q. Strategy to Construct Polyzwitterionic Hydrogel Coating with Antifouling, DragReducing and Weak Swelling Performance. RSC Adv. 2019, 9, 20812091.

(34) Xuan, Y.; Jiang, G.; Li, Y.; Yang, L.; Zhang, X. Biodegradable Oligo (Poly-l-Lysine) as a High-Performance Hydration Inhibitor for Shale. RSC Adv. 2015, 5, 84947-84958.

(35) Wang, J.; Zhao, Y.; Ma, F. X.; Wang, K.; Wang, F. B.; Xia, X. H. Synthesis of a Hydrophilic Poly-l-Lysine/Graphene Hybrid through Multiple Non-Covalent Interactions for Biosensors. J. Mater. Chem. B 2013, 1, 1406-1413.

(36) Movilli, J.; Di Iorio, D.; Rozzi, A.; Hiltunen, J.; Corradini, R.; Huskens, J. "Plug-n-Play" Polymer Substrates: Surface Patterning with Reactive-Group-Appended Poly-l-Lysine for Biomolecule Adhesion. ACS Appl. Polym. Mater. 2019, 1, 3165-3173.

(37) Han, Z.; Wang, Y.; Duan, X. Biofunctional Polyelectrolytes Assembling on Biosensors - A Versatile Surface Coating Method for Protein Detections. Anal. Chim. Acta 2017, 964, 170-177.

(38) Duan, X.; Mu, L.; Sawtelle, S. D.; Rajan, N. K.; Han, Z.; Wang, Y.; Qu, H.; Reed, M. A. Functionalized Polyelectrolytes Assembling on Nano-BioFETs for Biosensing Applications. Adv. Funct. Mater. 2015, 25, 2279-2286.

(39) Deming, T. J. Synthesis of Side-Chain Modified Polypeptides. Chem. Rev. 2016, 116, 786-808. 
(40) Movilli, J.; Rozzi, A.; Ricciardi, R.; Corradini, R.; Huskens, J. Control of Probe Density at DNA Biosensor Surfaces Using Poly(1Lysine) with Appended Reactive Groups. Bioconjugate Chem. 2018, 29, 4110-4118.

(41) Di Iorio, D.; Marti, A.; Koeman, S.; Huskens, J. Clickable Poly1-Lysine for the Formation of Biorecognition Surfaces. RSC Adv. 2019, 9, 35608-35613.

(42) Huang, C. J.; Li, Y.; Jiang, S. Zwitterionic Polymer-Based Platform with Two-Layer Architecture for Ultra Low Fouling and High Protein Loading. Anal. Chem. 2012, 84, 3440-3445.

(43) Masson, J. F.; Battaglia, T. M.; Davidson, M. J.; Kim, Y. C.; Prakash, A. M. C.; Beaudoin, S.; Booksh, K. S. Biocompatible Polymers for Antibody Support on Gold Surfaces. Talanta 2005, 67, 918-925.

(44) Masson, J. F. Surface Plasmon Resonance Clinical Biosensors for Medical Diagnostics. ACS Sens. 2017, 2, 16-30.

(45) Mullard, A. Cracking KRAS. Nat. Rev. Drug Discovery 2019, 18, $887-891$.

(46) Giesen, U.; Kleider, W.; Berding, C.; Geiger, A.; Orum, H.; Nielsen, P. E. A Formula for Thermal Stability (Tm) Prediction of PNA/DNA Duplexes. Nucleic Acids Res. 1998, 26, 5004-5006.

(47) Veerbeek, J.; Steen, R.; Vijselaar, W.; Rurup, W. F.; Korom, S.; Rozzi, A.; Corradini, R.; Segerink, L.; Huskens, J. Selective Functionalization with PNA of Silicon Nanowires on Silicon Oxide Substrates. Langmuir 2018, 34, 11395-11404.

(48) Turkevich, J.; Stevenson, P. C.; Hillier, J. A Study of the Nucleation and Growth Processes in the Synthesis of Colloidal Gold. Discuss. Faraday Soc. 1951, 11, 55-75.

(49) Shumaker-Parry, J. S.; Campbell, C. T. Quantitative Methods for Spatially Resolved Adsorption/Desorption Measurements in Real Time by Surface Plasmon Resonance Microscopy. Anal. Chem. 2004, 76, 907-917.

(50) Fischer, H.; Polikarpov, I.; Craievich, A. F. Average Protein Density Is a Molecular-Weight-Dependent Function. Protein Sci. 2004, 13, 2825-2828.

(51) Vörös, J. The Density and Refractive Index of Adsorbing Protein Layers. Biophys. J. 2004, 87, 553-561.

(52) Jung, L. S.; Campbell, C. T.; Chinowsky, T. M.; Mar, M. N.; Yee, S. S. Quantitative Interpretation of the Response of Surface Plasmon Resonance Sensors to Adsorbed Films. Langmuir 1998, 14, $5636-5648$.

(53) Allegretti, M.; Cottone, G.; Carboni, F.; Cotroneo, E.; Casini, B.; Giordani, E.; Amoreo, C. A.; Buglioni, S.; Diodoro, M.; Pescarmona, E.; Zazza, S.; Federici, O.; Zeuli, M.; Conti, L.; Cigliana, G.; Fiorentino, F.; Valle, M.; Giacomini, P.; Spinella, F. Cross-Sectional Analysis of Circulating Tumor DNA in Primary Colorectal Cancer at Surgery and during Post-Surgery Follow-up by Liquid Biopsy. J. Exp. Clin. Cancer Res. 2020, 39, No. 69.

(54) D’Agata, R.; Breveglieri, G.; Zanoli, L. M.; Borgatti, M.; Spoto, G.; Gambari, R. Direct Detection of Point Mutations in Nonamplified Human Genomic DNA. Anal. Chem. 2011, 83, 8711-8717.

(55) Xu, F.; Pellino, A. M.; Knoll, W. Electrostatic Repulsion and Steric Hindrance Effects of Surface Probe Density on Deoxyribonucleic Acid (DNA)/Peptide Nucleic Acid (PNA) Hybridization. Thin Solid Films 2008, 516, 8634-8639.

(56) D’Agata, R.; Giuffrida, M. C.; Spoto, G. Peptide Nucleic AcidBased Biosensors for Cancer Diagnosis. Molecules 2017, 22, No. 1951.

(57) McKeating, K. S.; Hinman, S. S.; Rais, N. A.; Zhou, Z.; Cheng, Q. Antifouling Lipid Membranes over Protein A for OrientationControlled Immunosensing in Undiluted Serum and Plasma. ACS Sens. 2019, 4, 1774-1782.

(58) D’Agata, R.; Bellassai, N.; Giuffrida, M. C.; Aura, A. M.; Petri, C.; Kögler, P.; Vecchio, G.; Jonas, U.; Spoto, G. A New Ultralow Fouling Surface for the Analysis of Human Plasma Samples with Surface Plasmon Resonance. Talanta 2021, 221, No. 121483.

(59) Halpern, A. R.; Wood, J. B.; Wang, Y.; Corn, R. M. SingleNanoparticle near-Infrared Surface Plasmon Resonance Microscopy for Real-Time Measurements of DNA Hybridization Adsorption. ACS Nano 2014, 8, 1022-1030.
(60) He, L.; Musick, M. D.; Nicewarner, S. R.; Salinas, F. G.; Benkovic, S. J.; Natan, M. J.; Keating, C. D. Colloidal Au-Enhanced Surface Plasmon Resonance for Ultrasensitive Detection of DNA Hybridization. J. Am. Chem. Soc. 2000, 122, 9071-9077.

(61) Xue, T.; Liang, W.; Li, Y.; Sun, Y.; Xiang, Y.; Zhang, Y.; Dai, Z.; Duo, Y.; Wu, L.; Qi, K.; Shivananju, B. N.; Zhang, L.; Cui, X.; Zhang, H.; Bao, Q. Ultrasensitive Detection of MiRNA with an AntimoneneBased Surface Plasmon Resonance Sensor. Nat. Commun. 2019, 10, No. 28.

(62) Krishnan, S.; Mani, V.; Wasalathanthri, D.; Kumar, C. V.; Rusling, J. F. Attomolar Detection of a Cancer Biomarker Protein in Serum by Surface Plasmon Resonance Using Superparamagnetic Particle Labels. Angew. Chem., Int. Ed. 2011, 50, 1175-1178.

(63) Kwon, M. J.; Lee, J.; Wark, A. W.; Lee, H. J. NanoparticleEnhanced Surface Plasmon Resonance Detection of Proteins at Attomolar Concentrations: Comparing Different Nanoparticle Shapes and Sizes. Anal. Chem. 2012, 84, 1702-1707.

(64) Kim, S.; Lee, H. J. Gold Nanostar Enhanced Surface Plasmon Resonance Detection of an Antibiotic at Attomolar Concentrations via an Aptamer-Antibody Sandwich Assay. Anal. Chem. 2017, 89, 6624-6630.

(65) Gifford, L. K.; Sendroiu, I. E.; Corn, R. M.; Lupták, A. Attomole Detection of Mesophilic DNA Polymerase Products by Nanoparticle-Enhanced Surface Plasmon Resonance Imaging on Glassified Gold Surfaces. J. Am. Chem. Soc. 2010, 132, 9265-9267.

(66) Krishnan, S.; Mani, V.; Wasalathanthri, D.; Kumar, C. V.; Rusling, J. F. Attomolar Detection of a Cancer Biomarker Protein in Serum by Surface Plasmon Resonance Using Superparamagnetic Particle Labels. Angew. Chem., Int. Ed. 2011, 50, 1175-1178.

(67) D’Agata, R.; Palladino, P.; Spoto, G. Streptavidin-Coated Gold Nanoparticles: Critical Role of Oligonucleotides on Stability and Fractal Aggregation. Beilstein J. Nanotechnol. 2017, 8, 1-11.

(68) Fathi, F.; Rashidi, M.-R.; Omidi, Y. Ultra-Sensitive Detection by Metal Nanoparticles-Mediated Enhanced SPR Biosensors. Talanta 2019, 192, 118-127.

(69) Wu, Y.; Tilley, R. D.; Gooding, J. J. Challenges and Solutions in Developing Ultrasensitive Biosensors. J. Am. Chem. Soc. 2019, 141, $1162-1170$.

(70) Lee, J. H.; Cho, H. Y.; Choi, H. K.; Lee, J. Y.; Choi, J. W. Application of Gold Nanoparticle to Plasmonic Biosensors. Int. J. Mol. Sci. 2018, 19, No. 2021.

(71) Schuck, P.; Zhao, H. The Role of Mass Transport Limitation and Surface Heterogeneity in the Biophysical Characterization of Macromolecular Binding Processes by SPR Biosensing. Methods Mol. Biol. 2010, 627, 15-54.

(72) Wan, J. C. M.; Massie, C.; Garcia-Corbacho, J.; Mouliere, F.; Brenton, J. D.; Caldas, C.; Pacey, S.; Baird, R.; Rosenfeld, N. Liquid Biopsies Come of Age: Towards Implementation of Circulating Tumour DNA. Nat. Rev. Cancer 2017, 17, 223-238.

(73) Holstein, C. A.; Griffin, M.; Hong, J.; Sampson, P. D. Statistical Method for Determining and Comparing Limits of Detection of Bioassays. Anal. Chem. 2015, 87, 9795-9801.

(74) Giuffrida, M. C.; Cigliana, G.; Spoto, G. Ultrasensitive Detection of Lysozyme in Droplet-Based Microfluidic Devices. Biosens. Bioelectron. 2018, 104, 8-14.

(75) Bellassai, N.; D’Agata, R.; Jungbluth, V.; Spoto, G. Surface Plasmon Resonance for Biomarker Detection: Advances in NonInvasive Cancer Diagnosis. Front. Chem. 2019, 7, No. 570. 